\title{
Efficient Hedging of Options with Probabilistic Haar Wavelets
}

\author{
Pedro José Catuogno, ${ }^{1}$ Sebastián Esteban Ferrando, ${ }^{2}$ \\ and Alfredo Lázaro González ${ }^{3}$ \\ ${ }^{1}$ Departamento de Matemática, Universidade Estadual de Campinas, 13.081-97 Campinas, SP, Brazil \\ ${ }^{2}$ Department of Mathematics, Ryerson University, 350 Victoria Street, Toronto, ON, Canada M5B 2K3 \\ ${ }^{3}$ Departamento de Matemática, Facultad de Ciencias Exactas y Naturales, \\ Universidad Nacional de Mar del Plata, Funes 3350, 7600 Mar del Plata, Argentina
}

Correspondence should be addressed to Sebastián Esteban Ferrando, ferrando@ryerson.ca

Received 29 March 2012; Accepted 19 April 2012

Academic Editors: M. Galea, J. Hu, and P. E. Jorgensen

Copyright (c) 2012 Pedro José Catuogno et al. This is an open access article distributed under the Creative Commons Attribution License, which permits unrestricted use, distribution, and reproduction in any medium, provided the original work is properly cited.

\begin{abstract}
The paper brings forward the issue of efficient representations of financial claims; in particular it addresses the problem of large transaction costs in hedging replications. Inspired by the localized properties of wavelets basis, Haar systems associated with space-time discretizations of continuous stochastic processes are proposed as a means to address the issue of efficient pathwise approximation. Theoretical developments are presented that justify the use of these approximations to construct self-financing portfolios by means of binary options. Upper bounds on the volume of transactions required to implement these portfolios are then established to illustrate the quality of the proposed approximations. The approach is applicable to general financial claims of European type, including path-dependent ones, for continuous underlying processes. Several numerical results and comparisons with delta hedging are also presented.
\end{abstract}

\section{Introduction}

From a mathematical perspective, hedging of financial contracts is a pathwise approximation that can also be realized by means of a financial portfolio. Constraints such as transactions costs and the discrete nature of hedging times, bring the question of efficient approximations upon us. In particular, existence of such approximations could make the markets more efficient. To the best of our knowledge, the issue of efficiency has, so far, not been addressed directly in the mathematical finance literature.

In delta hedging, the underlying is used to construct the portfolio replication; this involves an implicit linear spatial approximation, resulting from the Taylor expansion used in Ito's formula, for the value of the option. This approximate hedging gives a pointwise error the quality of which depends on the efficiency of this space-time approximation. 
The aim of the paper is to introduce new expansions that provide efficient hedging portfolio approximations to financial instruments. These approximations are naturally realizable via binary options and the notions of efficiency that we single out in our study are the number of transactions and the volume of transactions. This latter notion is defined as the total money exchanged during transactions required to set up the approximating hedging portfolio. These notions relate to the problem of transactions costs under the assumption that costs are proportional to the volume of transactions and/or there are costs related to the number of transactions (e.g., a fix cost per transaction). We express this symbolically below.

Definition 1.1. Given a path $\omega, \Pi_{1}$ and $\Pi_{2}$ two approximating hedging portfolios for a financial contract $X$, one will say that $\Pi_{1}$ is more efficient than $\Pi_{2}$ at $\omega$ if

$$
\left|\Pi_{1}-X\right| \leq\left|\Pi_{2}-X\right|, \quad \operatorname{VT}\left(\Pi_{1}\right)<\operatorname{VT}\left(\Pi_{2}\right), \# \Pi_{1}<\# \Pi_{2}
$$

holds at the given $\omega$, where $\operatorname{VT}\left(\Pi_{i}\right)(\omega)$ is the volume of transactions and $\# \Pi_{i}(\omega)$ the number of transactions, respectively, necessary to implement the portfolio $\Pi_{i}$ at $\omega$.

Clearly, the above definition can be easily modified to require the inequalities to hold with large probability or in the mean. It should be noted that the notion of volume of transactions is coordinate dependent, that is, it depends on the explicit approximation used to define the portfolios $\Pi_{i}$.

Our approach also allows to minimize the number of transactions required to implement the approximating portfolio, a small number makes the implementation of the portfolio realistic and reduces transaction costs when fixed costs are charged per transactions or they depend on the number of transactions in a laddered way.

Therefore, we aim to reduce the number and volume of transactions for a given approximation error; a key ingredient of our approach is the use of localized basic functions. The construction of our approximations is inspired by wavelet theory and nonlinear approximation theory (see [1, 2]), which studies efficient representations of functional classes. In the setup of the paper, we use the notion of $H$-system, an orthonormal set adapted to the given stochastic processes.

Reference [3] provides evidence of the high transaction costs incurred when performing frequent rebalancing in continuous models. Moreover, the volume of transactions required to implement delta hedging can be proved to diverge in a Black and Scholes model [4]. On the other hand, we describe next a related problem that occurs when European call options are used for hedging. For simplicity, consider an option $X$ that initiates at $t_{0}$ and expires at $T$ with $V_{t_{0}}(X)$ denoting the risk neutral price (or value) of $X$. Consider a digital option with payoff $X=\mathbf{1}_{S_{T} \geq K}$ and approximate this digital option with the following portfolio

$$
\Pi=\frac{1}{K_{2}-K_{1}}\left(X_{1}-X_{2}\right)
$$

where we go long on a European call $X_{1}=\left(S_{T}-K_{1}\right)_{+}$with strike $K_{1}$ and short on a European call $X_{2}=\left(S_{T}-K_{2}\right)_{+}$, with strike $K_{2}$ and $K_{1}<K<K_{2}$. We obtain a better and better approximation to $X$ by considering $\left(K_{2}-K_{1}\right) \rightarrow 0$. By no-arbitrage, it follows that 
$V_{t_{0}}(X) \approx V_{t_{0}}(\Pi)$ but the volume of transactions for $\Pi$ (which in this static example is a constant) is equal to

$$
V T(\Pi)=\frac{1}{K_{2}-K_{1}}\left[V_{t_{0}}\left(X_{1}\right)+V_{t_{0}}\left(X_{2}\right)\right]
$$

which can be arbitrarily large as $\left(K_{2}-K_{1}\right) \rightarrow 0$. Therefore, when decreasing the error of approximation we have the undesirable effect of increasing the volume of transactions. This is due to the fact that the approximation $X \approx \Pi$ is obtained by cancelation of (unbounded) terms and each term entering in this approximation will contribute separately to the volume of transactions. The example could be generalized to other payoffs containing a steep profile; the wavelet literature [5] points to the need to use localized approximating functions to handle singularities.

It is our contention that, in the two cases described above, the problem of large volume of transactions is created because both approximations cannot provide efficient approximations to some type of discontinuity present in the problem. By making use of binary options of the form $B=\mathbf{1}_{\left[t_{1}, T\right]} \mathbf{1}_{\left\{S_{t_{2} \in(a, b)}\right\}}$ (for given times $t_{1}<t_{2} \leq T$ ), conveniently structured in an $\mathrm{H}$-system, we prove that there exist financial portfolios built from these basic building blocks that provide small errors of approximation making use of few binary options and incurring in a low volume of transactions. In order to indicate the quality of our approximations, and as a contrast to existing hedging strategies, we provide bounds for the volume of transactions required to implement our approximating portfolios. We also provide several simulation examples; both the upper bounds and the numerical simulations provide excellent results. The upper bounds rely on the property of localization of our basis functions, this property implies that in order to approximate discontinuities only few localized basis functions are needed (at this point our approach is motivated by wavelet theory, [6, 7]). This is a crucial ingredient in order to achieve a small volume of transactions while using few transactions and achieving a small approximating error, see our Theorems 5.18 and 5.19 Some of these characteristics are in contrast with other approaches to hedging [8-10] which, similarly to our approach, use portfolios of simple options to hedge complex portfolios.

To indicate the essence of our approach, we point to (2.1), its right hand side is just a rewrite of the left hand-side in terms of the martingale differences that always form an orthogonal set. A novelty is in the writing of the conditional expectation as a Fourier expansion; the inner products $\left\langle X, u_{k}\right\rangle$ are a set of new coordinates with useful properties and information which can be used to achieve efficient approximations. Our expansions can be implemented by means of binary options; the idea of using binary options as providing a general approximation tool has been previously treated in [11]. Our approach relies on the use of linear combinations of binary options that have value zero at the time of entering the contracts. It is then important to limit their number and analyze the necessary volume of transactions to implement them so as to keep the transaction costs low. A more detailed explanation of the meaning of our approximations is presented at the opening of Section 4 .

The rest of the paper is organized as follows. Section 2 defines $H$-systems and develops some of the relationships between $H$-systems and sequences of partitions. Section 3 summarizes Willinger's main result on existence of discretization of stochastic processes and connects them to our setup of $H$-systems. Section 4 motivates and develops our main application to hedging a given European option. Section 5 presents several upper bounds for the volume of transactions required to implement the approximating portfolios described in Section 4. Section 6 presents several numerical examples and comparisons with delta 
hedging. Section 7 summarizes the main results of the paper. Appendix A introduces a simple example of an $H$-system in a basic financial setting. All the figures and tables mentioned in the paper present results from the numerical experiments. As a technical note, and for matters of convenience, we will suppress writing a.e. (almost everywhere) from many statements.

\section{H-Systems}

Let $(\Omega, \mathcal{A}, P)$ denote an arbitrary probability space. The notation \|\|$_{2}^{2}=\langle$,$\rangle stands for the$ inner product on $L^{2}(\Omega, \mathcal{A}, P)$ and \|\|$_{1}$ denotes the norm in $L^{1}$. The following Gundy's [12] definition is motivated by the standard Haar system on $L^{2}([0,1])$.

Definition 2.1. An orthonormal system of functions $\left\{u_{k}\right\}_{k \geq 0}$ defined on $\Omega$ is called an H-system if and only if for any $X \in L^{2}(\Omega, \mathcal{A}, P)$

$$
X_{A_{m}} \equiv \mathrm{E}\left(X \mid u_{0}, u_{1}, \ldots, u_{m}\right)=\sum_{k=0}^{m}\left\langle X, u_{k}\right\rangle u_{k}, \quad \forall m \geq 0,
$$

where $A_{m}=\sigma\left(u_{0}, \ldots, u_{m}\right)$. The intended meaning of $k \geq 0$ in the above definition is to allow the system $\left\{u_{k}\right\}_{k \geq 0}$ to be finite or infinite. We also use the notation $\mathscr{A}_{\infty}=\sigma\left(\cup_{m \geq 0} \mathcal{A}_{m}\right)$. In applications we will make use of the pointwise convergence of (2.1), which holds due to the martingale convergence theorem [13]. Moreover, if $p \in[1, \infty)$ is a given real number, then, for every $X \in L^{p}$, the sequence $X_{\mathscr{A}_{m}}=\mathrm{E}\left(X \mid \mathcal{A}_{m}\right)$ converges a.s. and in $L^{p}$ to $X_{\infty}=\mathbf{E}\left(X \mid \mathscr{A}_{\infty}\right)$. Convergence to $X$ holds whenever $\sigma(X) \subseteq \mathcal{A}_{\infty}$.

The following proposition, which is proven in [12], gives an alternative characterization of $H$-systems equivalent to Definition 2.1.

Proposition 2.2. An orthonormal system $\left\{u_{k}\right\}_{k \geq 0}$ defined on $\Omega$ is an $H$-system if and only if the following three conditions hold.

(1) Each $u_{k}$ assumes at most two nonzero values with positive probability.

(2) The $\sigma$-algebra $A_{m}$ consists exactly of $m+1$ elements.

(3) $\mathrm{E}\left(u_{k+1} u_{0}, u_{1}, \ldots, u_{k}\right)=0, k \geq 0$. So the functions $u_{k}$ are martingale differences.

Generating elements of discrete sigma algebras will be referred to as atoms.

Corollary 2.3. Assume that $\left\{u_{k}\right\}_{k \geq 0}$ is an $H$-system. Then, for each $n \geq 0, u_{n+1}$ takes two nonzero values (one positive and the other negative) only on one atom of $\mathcal{A}_{n}$ (hence this atom becomes its support). Consequently, $A_{n+1}$ consists of $n$ atoms from $A_{n}$ and two more atoms obtained by splitting the remaining atom from $\mathcal{A}_{n}$.

In view of the above proposition and its corollary, the functions in an $H$-system are natural generalizations of classic Haar functions, as the next definition states. 
Definition 2.4. Given $A \in \mathcal{A}, P(A)>0$, a function $\psi$ is called a Haar function on $A$ if there exist $A_{i} \in \mathcal{A}, A_{0} \cap A_{1}=\emptyset, A=A_{0} \cup A_{1}, \psi=a \mathbf{1}_{A_{0}}+b \mathbf{1}_{A_{1}}$, and

$$
\int_{\Omega} \psi(\omega) d P(\omega)=0, \quad \int_{\Omega} \psi^{2}(\omega) d P(\omega)=1
$$

Occasionally $\psi$ above will be denoted by $\psi_{A}$. We will attach the word Haar to several definitions and constructions related to $H$-systems.

\subsection{Basic Properties of H-Systems}

It should be clear, from Corollary 2.3, that an $H$-system naturally defines a binary tree of partitions, these are formally introduced in the next definition.

Definition 2.5. A sequence of partitions of $\Omega, R:=\left\{\mathcal{R}_{j}\right\}_{j \geq 0}$, is called a binary sequence of partitions if, for $j \geq 0$, the members of $\boldsymbol{R}_{j}$ have positive probability, $\boldsymbol{R}_{0}=\{\Omega\}$, and, for $j \geq 1$, $A \in \mathcal{R}_{j}$ if and only if it is also a member of $\mathcal{R}_{j-1}$ or, if not, there exists another member $A^{\prime}$ of $\mathcal{R}_{j}$, necessarily not in $\mathcal{R}_{j-1}$, such that $A \cup A^{\prime} \in \mathcal{R}_{j-1}$.

We set $A_{0,0}:=\Omega$, and hence $\mathcal{R}_{0}=\left\{A_{0,0}\right\}$. For $j \geq 1$, if $A \in \mathcal{R}_{j}$ and $A=A_{k, i} \in \mathcal{R}_{j-1}$ then $A$ preserves its index. Otherwise (i.e., $A \notin \mathcal{R}_{j-1}$, and not yet indexed) then there exists $A_{k, i} \in \mathcal{R}_{j-1}$ and $A^{\prime} \in \mathcal{R}_{j}$ such that

$$
A_{k, i}=A \cup A^{\prime}
$$

then set $A_{k+1,2 i}:=A$ and $A_{k+1,2 i+1}:=A^{\prime}$. In that case we will say " $A_{k, i}$ splits in $\mathcal{R}_{j}$ ".

The index $k$ in $A_{k, i}$ will be called the scale parameter (we will also call it the level), it indicates the number of times $A_{0,0}$ has been split to obtain $A_{k, i}$. Notice that $\mathcal{R}_{j}$ can have at most $2^{j}$ members, and if $A_{k, i} \in \mathcal{R}_{j}$ then $k \leq j$ and $0 \leq i \leq 2^{k}-1$. Observe also that $\boldsymbol{R}_{j}$ refines $\boldsymbol{R}_{j-1}$. The tree shown in Figure 7 illustrates this indexation, it corresponds to an $H$-system with $m=5$.

Observe that $A_{3,2}, A_{3,3}, A_{3,4}$, and $A_{3,5}$ do not appear, the reason being that $A_{2,1}$ and $A_{2,2}$ do not split.

Given a binary sequence of partitions $\left\{\boldsymbol{R}_{j}\right\}_{j \geq 0}$, define the associated trees

$$
\tau_{n} \equiv \cup_{j=0}^{n} \mathcal{R}_{j}, \quad \tau \equiv \cup_{n \geq 0} \tau_{n}
$$

The parent-child relationship is given by the split relationship. For every splitting internal node $A \in \tau$, we have, using the indexation introduced in Definition 2.5 ( $A=A_{k, i}=A_{k+1,2 i} \cup$ $\left.A_{k+1,2 i+1}\right)$, a corresponding Haar function at that node

$$
\psi_{k, i}=a_{k, i} \mathbf{1}_{A_{k+1}, 2 i}+b_{k, i} \mathbf{1}_{A_{k+1}, 2 i+1}
$$


where, according to Definition 2.4,

$$
a_{k, i}=\frac{\sqrt{P\left(A_{k+1,2 i+1}\right)}}{\sqrt{P\left(A_{k, i}\right) P\left(A_{k+1,2 i}\right)}}, \quad b_{k, i}=\frac{-\sqrt{P\left(A_{k+1,2 i}\right)}}{\sqrt{P\left(A_{k, i}\right) P\left(A_{k+1,2 i+1}\right)}} .
$$

Given $A \in \tau$, we have the natural associated tree $\tau_{A}$ with root $A$.

The following sets of indexes will be used throughout the paper, and in Appendix A, consider $j \geq 0$ and let

$$
\begin{gathered}
H_{j} \equiv\left\{(k, i): A_{k, i} \in \mathcal{R}_{j}, A_{k, i}=A_{k+1,2 i} \cup A_{k+1,2 i+1} \text { with } A_{k+1,2 i}, A_{k+1,2 i+1} \in \mathcal{R}_{j+1}\right\}, \\
K_{j} \equiv\left\{(k, i): A_{k, i} \in \mathcal{R}_{j}\right\} .
\end{gathered}
$$

$H_{j}$ collects the indices of members of $\boldsymbol{R}_{j}$ that split in $\boldsymbol{R}_{j+1}$, those are going to be the support of the Haar functions. In the tree illustration shown in Figure 7 we have $K_{2}=$ $\{(2,0),(2,1),(2,2),(2,3)\}, H_{2}=\{(2,0),(2,3)\}, K_{3}=\{(3,0),(3,1),(2,1),(2,2),(3,6),(3,7)\}$ and $H_{3}$ not defined.

For each $A_{k, i} \in \mathcal{R}_{j}$, let

$$
\phi_{k, i} \equiv \frac{\mathbf{1}_{A_{k, i}}}{\sqrt{P\left(A_{k, i}\right)}}
$$

$\left\{\phi_{k, i}\right\}_{(k, i) \in K_{j}}$ is an orthonormal basis of $L^{2}\left(\Omega, \sigma\left(\mathcal{R}_{j}\right), P\right)$. Under the assumption that all atoms in $\mathcal{R}_{j}$ split, the present notation relates easily to the abstract definition in (2.1), namely, given a binary sequence of partitions $\left\{\mathcal{R}_{j}\right\}_{j \geq 1}$, there is associated the $H$-system given by $u_{0}=\phi_{0,0} \equiv 1_{\Omega}$ and $\left\{u_{2^{k}+i} \equiv \psi_{k, i}:(k, i) \in H_{j}, j \geq 1,\right\}$. In general the following expansion holds for any $H_{-}$ system:

$$
\begin{aligned}
X_{\mathcal{R}_{j}} \equiv \mathrm{E}\left(X \mid \sigma\left(\mathcal{R}_{j}\right)\right)(\omega) & =\left\langle X, \phi_{0,0}\right\rangle \phi_{0,0}+\sum_{m=0}^{j-1} \sum_{(k, i) \in H_{m}}\left\langle X, \psi_{k, i}\right\rangle \psi_{k, i} \\
& =\sum_{(k, i) \in K_{j}}\left\langle X, \phi_{k, i}\right\rangle \phi_{k, i} .
\end{aligned}
$$

Therefore, one has the following result (the formal proof and more details are provided in [14].)

Theorem 2.6. Every H-system induces naturally a binary sequence of partitions and reciprocally.

We will use $H$-systems to approximate stochastic processes, a finite partition of the time interval $[0, T]$ will provide a finite $H$-system, and each atom $A$ in the associated tree $\tau_{n}$ will correspond to an event in the space-time discretization of the process. Section 3 describes general results that construct these space-time approximations that provide pointwise convergence to a given continuous stochastic process. These results will guarantee the availability of $H$-systems for further developments in the paper. 


\section{Discretizations of Stochastic Processes}

In order to make use of Definition 2.1 and Theorem 2.6 we will need results establishing the existence of discrete approximations of continuous stochastic processes. The main result is presented in Proposition 3.4, it gives an existence result that can be employed in our applications. Alternative constructions of $H$-systems are presented in [14]. Reference [15] describes ways to construct $H$-systems associated with nested partitions.

Let $(\Omega, \mathcal{A}, P)$ be a complete probability space and $S=\left(S_{t}: 0 \leq t \leq T\right)$ be a continuous stochastic process defined on this probability space. Let $\mathcal{F}=\left\{\mathcal{F}_{t}: 0 \leq t \leq T\right\}$ be the filtration where $\mathcal{F}_{t}$ is the completion of $\sigma\left(S_{r}: 0 \leq r \leq t\right)$. Following Willinger and Taqqu $[16,17]$, we introduce the notion of skeleton-approach for stochastic processes.

Definition 3.1. A continuous-time skeleton approach of $S$ is a triple $\left(I^{\xi}, \mathcal{F}^{\xi}, \xi\right)$, consisting of an index-set $I^{\xi}$, a filtration $\mathcal{F}^{\xi}=\left\{\mathscr{F}_{t}^{\xi}: 0 \leq t \leq T\right\}$, the skeleton filtration, and a $\mathcal{F}^{\xi}$-adapted process $\xi=(\xi: 0 \leq t \leq T)$ such that it verifies the following

(1) $I^{\xi}=\left\{0=t(\xi, 0)<\cdots<t\left(\xi, N_{\xi}\right)=T\right\}$, where $N_{\xi}<\infty$.

(2) For each $t, \mathcal{F}_{t}^{\xi}$ is a finitely generated sub- $\sigma$-algebra of $\mathcal{F}_{t}$, with atomic partition $p_{t}^{\xi}$.

(3) For $t \in[0, T]-I^{\xi}$, one set $\mathcal{F}_{t}^{\xi}=\mathcal{F}_{t(\xi, k)}^{\xi}$ if $t \in[t(\xi, k), t(\xi, k+1))$ for some $0 \leq k<N_{\xi}$.

(4) For each $0 \leq t \leq T, \xi_{t}=\mathbf{E}\left(S_{t} \mid \mathscr{F}_{t}^{\xi}\right)$.

Definition 3.2. A sequence $\left(I^{n}, \mathcal{F}^{n}, \xi^{n}\right)$ of continuous time skeletons of $S$ will be called a continuous-time skeleton approximation of $S$ if the following three properties hold. The sequence $I^{n}$ of indexes satisfies

$$
\lim _{n \rightarrow \infty}\left|I^{n}\right|=0,
$$

where $\left|I^{n}\right| \equiv \max \left\{\left|t\left(\xi^{n}, k\right)-t\left(\xi^{n}, k-1\right)\right|: 1 \leq k \leq N_{n}\right\}$, and $I \equiv \cup_{n} I^{n}$ is a dense subset of $[0, T]$. For each $0 \leq t \leq T$,

$$
\begin{aligned}
& \mathcal{F}_{t}^{n} \uparrow \mathcal{F}_{t}, \\
& P\left(\left\{\omega \in \Omega: \lim _{n \rightarrow \infty} \sup _{0 \leq t \leq T}\left|S_{t}(\omega)-\xi_{t}^{n}(\omega)\right|=0\right\}\right)=1 \text {. }
\end{aligned}
$$

The fundamental result of Willinger ([16] pp 55, Lemma 4.3.1) is stated next, it guarantees the existence of continuous-time skeleton approximations for continuous processes. These discrete pathwise approximations are finite in space and time.

Lemma 3.3. Assume $S$ that is a continuous process, then there exists a continuous-time skeleton approximation for $S$.

The atoms in Willinger's construction are given by intersections of sets of the form $S_{t_{i}}^{-1}(a, b)$ corresponding to different times $t_{j}$. Therefore, the atoms are natural events associated with level sets of the underlying process $S_{t}$. 
Each continuous time skeleton $\left(I^{\xi}, \mathcal{F}^{\xi}, \xi\right)$ of $S$ determines a sequence of nested finite partitions $\left\{\boldsymbol{D}_{t_{s}}^{\xi}\right\}_{s=0}^{N_{\xi}}$. Clearly, there exists a binary sequence of partitions $\left\{\mathcal{R}_{j}^{\xi}\right\}_{j \geq 0}$, and a finite sequence of indexes $0=j_{0}<j_{1}<\cdots<j_{N_{\xi}}$ such that

$$
R_{j_{s}}^{\xi}=p_{t_{s}}^{\xi} \quad \text { for } s=0, \ldots, N_{\xi}
$$

Indeed, let $\mathcal{R}_{0}^{\xi} \equiv\{\Omega\}$, and assume that $R_{j}^{\xi}$, for $j \leq j_{s}$, has been constructed and indexed as in Definition 2.5. then we may construct $R_{j}^{\xi}$ for $j>j_{s}$ in the following way: first, include in $R_{j_{s}+1}^{\xi}$ all those members of $R_{j_{s}}^{\xi}\left(=D_{t_{s}}^{\xi}\right)$ that are also atoms of $D_{t_{s+1}}^{\xi}$ (these are events that do not undergo a split in the period $\left[t_{s}, t_{s+1}\right)$.) Next, for each $A_{k, i} \in \mathcal{R}_{j_{s}}^{\xi}$, which can be written as a union of $B_{1}, \ldots, B_{m} \in P_{t_{s+1}}^{\xi}(m>1)$, set $A_{k+1,2 i}:=\cup_{j=0}^{[m / 2]} B_{j}$ and $A_{k+1,2 i+1}:=\cup_{[m / 2]+1}^{m} B_{j}$ in $\mathcal{R}_{j_{s}+1}^{\xi}$. Continue this process, changing the role of $R_{j_{s}}^{\xi}$ by $R_{j_{s}+1}^{\xi}$, up to some $j_{s+1}$ satisfying $R_{j_{s+1}}^{\xi}=$ $p_{t_{s+1}}^{\xi}$. Therefore, as an application of Theorem 2.6 to the binary sequence of partitions $\left\{\mathcal{R}_{j}^{\xi}\right\}_{j \geq 0}$ described above, we obtain a finite $H$-system associated with each continuous time skeleton $\left(I^{\xi}, \mathcal{F}^{\xi}, \xi\right)$ of $S$. Clearly, these $H$-systems are adapted to the filtration $\mathcal{F}_{t_{s}}^{\xi}$, that is, $\psi_{k, i} \in \mathcal{F}_{t_{s}}^{\xi}$ for $(k, i) \in H_{j}$ with $j<j_{s}$.

Proposition 3.4. Let $\mathcal{F}=\left\{\mathcal{F}_{t}: 0 \leq t \leq T\right\}$ be the filtration where $\mathcal{F}_{t}$ is the completion of $\sigma\left(S_{r}: 0 \leq\right.$ $r \leq t)$. Then there exist a sequence of finite $H$-systems $\left(\mathscr{\ell}^{n}=\left\{\mathbf{1}_{\Omega}, \psi_{k, i}^{n}\right\}\right)$ and two sequences of finite indexes $\left(I^{n}=\left\{0=t_{0}^{n}<\cdots<t_{N_{n}}^{n}=T\right\}\right)$ and $\left(J^{n}=\left\{0=j_{0}^{n}<\cdots<j_{N_{n}}^{n}\right\}\right)$ such that

(1) $\psi_{k, i}^{n} \in \mathcal{F}_{t}^{n}$ for $(k, i) \in H_{j}$ with $j<j_{s}^{n}$,

(2) for each $0 \leq t \leq T$,

$$
\lim _{n \rightarrow \infty} \sup \left\{S_{t}-\xi_{t}^{n}: 0 \leq t \leq T\right\}=0 \quad \text { a.e., }
$$

where $\xi_{t}^{n}=\mathbf{E}\left(S_{t}\right) \mathbf{1}_{\Omega}+\sum_{j=0}^{j_{s}^{n}-1} \sum_{(k, i) \in H_{j}}\left\langle S_{t}, \psi_{k, i}^{n}\right\rangle \psi_{k, i}^{n}$ for $t \in\left[t_{s-1}^{n}, t_{s}^{n}\right)$.

Proof. Let $\left(I^{n}, \Psi^{n}, \xi^{n}\right)$ be a continuous-time skeleton approximation of $S$. Using Theorem 2.6 construct for each $n$ an $H$-system $\left(\mathscr{L}^{n}=\left\{\mathbf{1}_{\Omega}, \psi_{j, i}^{n}\right\}\right)$ associated with the sequence of partitions $\left\{R_{j}^{n}\right\}_{j \geq 0}$ satisfying (3.3). In order to conclude the proof it is sufficient to observe that, in virtue of the main property of $H$-systems, (2.1), $\xi_{t}^{n}=\mathbf{E}\left(S_{t} \mid \mathcal{F}_{t}^{n}\right)=\mathbf{E}\left(S_{t}\right) \mathbf{1}_{\Omega}+$ $\sum_{j=0}^{j_{s}^{n}-1} \sum_{(k, i) \in H_{j}}\left\langle S_{t}, \psi_{k, i}^{n}\right\rangle \psi_{k, i}^{n}$ for $t \in\left[t_{s-1}^{n}, t_{s}^{n}\right)$.

To be clear, we briefly remark on the connection between Proposition 3.4 and (2.1). The functions $u_{k}$ appearing in (2.1) are the functions $\psi_{k, i}^{n}$ introduced in Proposition 3.4, the key remark being the extra parameter $n$ that corresponds to the time interval discretizations $I^{n}$. Once more, we rewrite (2.1) in the notation of the present section,

$$
\begin{aligned}
\mathbf{E}\left(X \mid \sigma\left(p_{t_{s}}^{n}\right)\right) & =\mathbf{E}\left(X \mid \sigma\left(\mathcal{R}_{j_{s}}^{n}\right)\right)=\mathbf{E}\left(X \mid\left\{\mathbf{1}_{\Omega}, \psi_{k, i}^{n}\right\}_{(k, i) \in H_{j}, 0 \leq j<j_{s}}\right) \\
& =\mathbf{E}(X) \mathbf{1}_{\Omega}+\sum_{j=0}^{j_{s}-1} \sum_{(k, i) \in H_{j}}\left\langle X, \psi_{k, i}^{n}\right\rangle \psi_{k, i^{\prime}}^{n} \quad \forall s=0, \ldots, N_{n} .
\end{aligned}
$$


The sigma algebra $\mathcal{A}_{m}$, appearing in (2.1), in the present context equals $\mathcal{A}_{m}=\mathcal{A}_{m_{s}} \equiv$ $\sigma\left(\left\{\psi_{k, i}^{n}\right\}_{(k, i) \in H_{j}, 0 \leq j<j_{s}}\right)=\sigma\left(\mathcal{D}_{t_{s}}^{n}\right)$ (where $m_{s}$ is the cardinality of the set $\left\{\psi_{k, i}^{n}\right\}_{(k, i) \in H_{j}, 0 \leq j<j_{s}}$.) Notice also that

$$
\mathrm{E}\left(X \mid \sigma\left(\mathcal{R}_{j_{s}}^{n}\right)\right)=\mathbf{E}\left(X \mid \sigma\left(\mathcal{R}_{j_{s-1}}^{n}\right)\right)+\sum_{j=j_{s-1}(k, i) \in H_{j}}^{j_{s}-1}\left\langle X, \psi_{k, i}^{n}\right\rangle \psi_{k, i^{\prime}}^{n} \quad \forall s=0, \ldots, N_{n}
$$

\section{Hedging Portfolios of Binary Options}

This section illustrates how $H$-systems can be applied in financial mathematics. It develops briefly a theory of hedging based on binary options, the martingale property of the $H$-system is put to use in this theory.

Let us first discuss the main idea of how the developments in the present section address the problem of high volume of transactions described in Section 1. First note that $u_{0}=\phi_{0,0}=\mathbf{1}_{\Omega}$ and, so, this function can be implemented by means of a bank account, the Haar functions are of the form $\psi_{k, i}=a_{k, i} \mathbf{1}_{A_{k+1,2 i}}+b_{k, i} \mathbf{1}_{A_{k+1,2 i+1}}$, which under natural conditions can be realized by means of binary options (involving short selling). Recall that $\int_{\Omega} \psi_{k, i} d P=0$ and the support of $\psi_{k, i}$ is $A_{k, i}$, which is the disjoint union of $A_{k+1,2 i}$ and $A_{k+1,2 i+1}$. It is clear that $\left\langle X, \psi_{k, i}\right\rangle \psi_{k, i}$ approximates the oscillations of $X-\mathbf{E}_{A_{k, i}}(X)$ on $A_{k, i}$, where $\mathbf{E}_{A_{k, i}}(X)$ denotes the expectation on $A_{k, i}$. In general, the events $A_{k+1,2 i}$ and $A_{k+1,2 i+1}$ will be level sets of financially relevant random variables, and hence the function $\psi_{k, i}$ captures fluctuations in $X$ due to these two financial events. In short, the financial meaning of (2.1) is the use of the bank account to capture the mean value of $X$ and the use of binary options to capture the (local) oscillations of $X$ about this (local) mean value. Even though Haar hedging uses (binary) options to build the replicating portfolio, it will be misleading to call it a static [9] type of hedging as we explain next. In general, each $\psi_{k, i}$ is localized to its support, say the atom $A_{k, i}$, this atom will be localized in time to same interval $\left[s_{a}, t_{a}\right]$ (essentially, this means that $A_{k, i}$ is generated by the random variables $\left\{S_{t}\right\}_{S_{a} \leq t \leq t_{a}}$ ) and will also be localized in space (it will be the level set of some appropriate random variable). This localization of the Haar functions, and hence of the binary options, has the effect that for a given unfolding path $w \in \Omega$ only the Haar functions in (2.1) whose support contain this $w$ have to be implemented by the Haar hedging portfolio. This is the essence of dynamic hedging.

In short, the left-hand side of (2.1) is a martingale that, under appropriate conditions, approximates $X$ a.e. In this way, we have a sequence of portfolios of binary options converging a.e. to $X$. Moreover these portfolios can be implemented dynamically, via financial transactions, in a self-financing way due to the martingale property. Therefore, we have available a discrete, self-financing, hedging strategy to approximate $X$. This hedging strategy will be referred to as Haar hedging next.

\subsection{Formal Developments}

For the sake of simplicity, we will work in a market model $\left(\Omega, \mathcal{F},\left(\mathcal{F}_{t}\right)_{0 \leq t \leq T}, P\right)$ with the usual assumptions; we refer to [18] for background. Let $B=\left(B(t)=e^{r t}\right)$ be the bond and a nonnegative adapted continuous stochastic process $S=\left(S_{t}\right)_{0 \leq t \leq T}$ the price process. As in Section 3, $\mathcal{F}_{t}$ is the completion of $\sigma\left(S_{r}: 0 \leq r \leq t\right)$. We assume that $P$ is the risk neutral measure, that is, the discounted price process $\left(e^{-r(T-t)} S_{t}\right)$ is a martingale. Let $\left(I^{n}, \mathcal{F}^{n}, \xi^{n}\right)$ be a 
continuous-time skeleton approximation of $S$. For fixed $n$, let $\mathcal{R}^{n}=\left\{\mathcal{R}_{j}^{n}\right\}_{j \geq 0}$ be a sequence of binary partitions associated with the sequence of partitions $\left\{D_{t_{s}}^{n}\right\}_{s=0}^{N_{n}}$ as described in Section 3 (see (3.3)), associated, via Theorem 2.6, with the $H$-system $\left\{\mathbf{1}_{\Omega}, \psi_{k, i}^{n}\right\}$ defined on $\Omega$. We also assume that a European derivative $X \in L^{1}\left(\Omega, \sigma\left(\cup_{n \geq 0} \mathcal{F}_{T}^{n}\right), P\right)$ is given. In the remaining of this section we will drop the index $n$ as it will be fixed in all computations. The notation $V_{t}(Z)$ will denote the risk neutral value process of an instrument $Z$, in our setup we have $V_{t}(Z)=e^{-r(T-t)} \mathbf{E}\left(Z \mid \mathcal{F}_{t}\right)$.

As a sufficient condition for the atoms in a binary sequence of partitions to be used in a dynamic hedging portfolio we will impose a natural association between the martingale property of the $H$-system and a sequence of rebalancing times. In particular, in order to define dynamic hedging strategies, we will use the concept of time support of events.

Definition 4.1. Let $E \in \mathcal{F}_{T}$, we say that $E$ is localized to the time interval $[a, b]$, or that the time support of $E$, denoted by $t-\operatorname{supp}(E)$ is contained in $[a, b]$ if $E \in \sigma\left(S_{t}, t \in[a, b]\right)$. Also, let $t-\operatorname{supp}(E)$ denote the intersection of all such intervals $[a, b]$.

The following definition is an extension to partitions of the notion of time localization of events.

Definition 4.2. Let $D \subset \mathcal{F}_{T}$ be a partition of $\Omega . P$ is said to be localized to the time interval $[a, b]$ if each $B \in P$ is localized to $[a, b]$. Moreover, define the $t-\operatorname{supp}(D)$ as the intersection of the all intervals $[a, b]$ such that $D$ is localized to that interval.

The definition below is the cornerstone of our dynamic hedging strategy based on $H$-systems.

Definition 4.3. Let $R=\left\{\mathcal{R}_{j}\right\}_{0 \leq j \leq J}$ be a sequence of binary partitions, we say that $R$ is localized to the time sequence $t_{0}=0<\cdots<t_{n}=T$ if there exists a sequence $j_{1}<\cdots<j_{n}=J$ such that $t-\operatorname{supp}\left(\mathcal{R}_{j_{s}}\right) \subset\left[t_{0}, t_{s}\right]$ for $s=1, \ldots, n$. We call the sequence $j_{1}, \ldots, j_{n}$ the levels of localization of $R$.

The financial blocks underlying $\mathcal{R}$ are the binary options

$$
\mathbf{B}_{k, i}=\left(\mathbf{1}_{A_{k, i}}(t) \equiv \mathbf{1}_{\left[t_{s}, T\right]}(t) \mathbf{1}_{A_{k, i}}\right), \quad(k, i) \in K_{j}, \text { for } j_{s}<j \leq j_{s+1},
$$

which are acquired at time $t_{s-1}$ and reach their maturity at time $t_{s}$. These binary options have payoff $\mathbf{1}_{A_{k, i}}$ at time $t_{s}$.

To have a financial realization of the hedging we are proposing, we need to assume $\mathcal{R}$ to be admissible as defined in the next definition.

Definition 4.4 (assumption on financial realization). The binary sequence of partitions $\mathcal{R}$ is called admissible if for any integer $j$ and each atom $A_{k, i} \in \mathcal{R}_{j}$ the binary options $\mathbf{B}_{k, i}$ are available for trading, in particular, short selling is possible.

In order to simplify the exposition, when defining the Haar hedging portfolio below, we will further define the Haar obligations as follows: $\Psi_{k, i}$, where $\Psi_{k, i}(t) \equiv \mathbf{1}_{\left[t_{s}, T\right]}(t) \psi_{k, i}=$ $\mathbf{1}_{\left[t_{s}, T\right]}(t)\left(a_{k, i} \mathbf{1}_{A_{k+1,2 i}}+b_{k, i} \mathbf{1}_{A_{k+1,2 i+1}}\right)=a_{k, i} \mathbf{B}_{k+1,2 i}+b_{k, i} \mathbf{B}_{k+1,2 i+1}$, with $(k, i) \in H_{j}, j_{s} \leq j<j_{s+1}$ which are obligations at time $t_{s}$ that are acquired at time $t_{s-1}$, for each $s=1, \ldots, N$. At no point in the 
paper we require that the Haar obligations exist as financial instruments, they are financially realized only in terms of the binary options.

Remark 4.5. Next we will define two hedging strategies via self-financing portfolios that approximate a European option using $H$-systems. In fact, we introduce the strategy НП associated with Haar obligations and the strategy ВП associated to binary options. These two portfolios will be used in such a way that the two will give exactly the same approximations at all times (see Section 4.2), The difference being that they will be implemented through different binary options and hence will have different volume of transactions.

\section{Haar Hedging Portfolio}

$\mathrm{H} \Pi_{\mathcal{R}}(X)=\left(\mathrm{H} \Pi_{\mathcal{R}}(X)_{t}\right)$ will be a predictable, vector-valued, stochastic processes constant on the intervals $t_{s-1} \leq t<t_{s}$. The portfolio $\mathrm{H}_{\mathcal{R}}(X)_{t}$ is rebalanced at times $t_{s-1}$ replicating $e^{-r\left(T-t_{s}\right)} \mathbf{E}\left(X \mid \sigma\left(\mathcal{R}_{j_{s}}\right)\right)$ for $s=1, \ldots, N-1$. As previously indicated, this portfolio approximates fluctuations of the option about its mean value by means of the Haar functions. Taking $N=1$ the construction gives, as a special case, an example of static hedging. At each time $t_{s-1}$ we will specify how much to invest in the bond and how much to invest in the Haar obligations available at that rebalancing time, this will specify the coordinates of the vector $\mathrm{H}_{\mathcal{R}}(X)_{t}$. Here are the coordinates of $\mathrm{H} \Pi_{\mathcal{R}}(X)_{t}$ for $t \in\left[t_{0}, t_{1}\right)$

$$
\begin{gathered}
e^{-r\left(T-t_{0}\right)} \mathbf{E}(X) \quad \text { invested in the bond and } \\
e^{-r\left(T-t_{1}\right)}\left\langle X, \psi_{k, i}\right\rangle \quad \text { invested in } \Psi_{k, i} j=0, \ldots j_{1}-1,(k, i) \in H_{j} .
\end{gathered}
$$

The coefficients $\left\langle X, \psi_{k, i}\right\rangle$ correspond to the expansion of $\mathbf{E}\left(X \mid \sigma\left(D_{t_{s}}\right)\right)$ given in (3.5). As a short hand notation we will use

$$
x_{k}[i] \equiv \mathbf{E}\left(X \mid \sigma\left(\mathcal{R}_{j}\right)\right)(w)=\frac{1}{P\left(A_{k, i}\right)} \int_{A_{k, i}} X d P, \quad \omega \in A_{k, i}, \text { for }(k, i) \in K_{j} .
$$

Observe that the purchasing value of this portfolio is $V_{t_{0}}\left(H \Pi_{\mathcal{R}}(X)\right)=e^{-r\left(T-t_{0}\right)} \mathbf{E}(X)$. The following (inductive) step will be to rebalance the portfolio at time $t_{s-1}$. Assume that at this time we are in the event $A_{k_{0}, i_{0}}$ with $\left(k_{0}, i_{0}\right) \in K_{j_{s-1}}$, and the value of this portfolio is $e^{-r\left(T-t_{s-1}\right)} x_{k_{0}}\left[i_{0}\right]$.

The rebalanced coordinates of $\mathrm{H}_{\mathcal{R}}(X)_{t}$ for $t \in\left[t_{s-1}, t_{s}\right)$ are

$$
\begin{aligned}
& e^{-r\left(T-t_{s-1}\right)} x_{k_{0}}\left[i_{0}\right] \quad \text { invested in the bond and } \\
& e^{-r\left(T-t_{s}\right)}\left\langle X, \psi_{k, i}\right\rangle \quad \text { invested in } \Psi_{k, i},(k, i) \in H_{j}^{k_{0}, i_{0}}, j=j_{s-1}, \ldots j_{s}-1,
\end{aligned}
$$

where $H_{j}^{k_{0}, i_{0}} \equiv\left\{(k, i) \in H_{j}: A_{k, i} \subseteq A_{k_{0}, i_{0}}\right\}\left(H_{j}^{k_{0}, i_{0}}\right.$ could be empty if $A_{k_{0}, i_{0}}$ does not split at time $\left.t_{s}\right)$. Recall that the obligations $\Psi_{k, i}$ involved expire at time $t_{s}$.

Theorem 4.6. Under the assumption $V_{t_{s}}\left(\Psi_{k, i}\right)=0$, for all $(k, i)$ and all $s=1, \ldots, n-1$, the portfolio $H \Pi_{\mathcal{R}}(X)_{t}$ is self-financing and replicates $e^{-r\left(T-t_{s}\right)} \mathbf{E}\left(X \mid \sigma\left(\mathcal{R}_{j_{s}}\right)\right)$ at $s=1, \ldots, N$. 
Proof. We proceed by induction on $s$. For $s=1$ the portfolio $H \Pi_{\mathcal{R}}(X)_{t}$ is given by (4.2) when $t \in\left[t_{0}, t_{1}\right)$. It is clear from (3.5) that $H \Pi_{\mathcal{R}}(X)_{t_{0}}$ replicates $e^{-r\left(T-t_{1}\right)} \mathbf{E}\left(X \mid \sigma\left(\mathcal{R}_{j_{1}}\right)\right)$ and is selffinancing because $V_{t_{0}}\left(H \Pi_{\mathcal{R}}(X)_{t_{0}}\right)=e^{-r\left(T-t_{0}\right)} \mathbf{E}(X)$ since $\mathbf{E}\left(\psi_{j, i}\right)=0$.

For convenience, we will use the notation $t^{-}=t-\epsilon, \epsilon>0$. For the inductive step, at time $t_{s-1}$ the process is in some event $A_{k_{0}, i_{0}}$ with $\left(k_{0}, i_{0}\right) \in K_{j_{s-1}}$ and assume

$$
V_{t_{s-1}}\left(H \Pi_{\mathcal{R}}(X)_{t_{s-1}^{-}}\right)(\omega)=e^{-r\left(T-t_{s-1}\right)} \mathbf{E}\left(X \mid \sigma\left(\mathcal{R}_{j_{s-1}}\right)\right)(\omega)=e^{-r\left(T-t_{s-1}\right)} x_{k_{0}}\left[i_{0}\right]
$$

for $\omega \in A_{k_{0}, i_{0}}$. The re-balancing of $H \Pi_{\mathcal{R}}(X)_{t}$ at $t_{s-1}$ is given by (4.4), for all $t \in\left[t_{s-1}, t_{s}\right)$; the cases of $A_{k_{0}, i_{0}}$ splitting at the next level or not are simultaneously handled by the cases $H_{j}^{k_{0}, i_{0}} \neq \emptyset$ or $H_{j}^{k_{0}, i_{0}}=\emptyset$, respectively. The purchasing of $H \Pi_{\mathcal{R}}(X)_{t_{s-1}}$ is self-financing since the value of the portfolio given by (4.4) is $e^{-r\left(T-t_{s-1}\right)} x_{k_{0}}\left[i_{0}\right]$, this follows from our assumption that $V_{t_{s-1}}\left(\Psi_{k, i}\right)=0$. Considering $t=t_{s}$, by (3.6), (4.4), and (3.5), we compute

$$
\begin{aligned}
V_{t_{s}}\left(H \Pi_{\mathcal{R}}(X)_{t_{s}^{-}}\right)= & e^{-r\left(T-t_{s-1}\right)} x_{k_{0}}\left[i_{0}\right] e^{r\left(t_{s}-t_{s-1}\right)} \mathbf{1}_{A_{k_{0}, i_{0}}} \\
& +e^{-r\left(T-t_{s}\right)} \sum_{j=j_{s-1}}^{j_{s}-1} \sum_{(k, i) \in H_{j}^{k_{0}, i_{0}}}\left\langle X, \Psi_{k, i}\right\rangle V_{t_{s}}\left(\Psi_{k, i}\left(t_{s}\right)\right) \\
= & e^{-r\left(T-t_{s}\right)} x_{k_{0}}\left[i_{0}\right] \mathbf{1}_{A_{k_{0}, i_{0}}}+e^{-r\left(T-t_{s}\right)} \sum_{j=j_{s-1}(k, i) \in H_{j}^{k_{0}, i_{0}}}^{j_{s}-1}\left\langle X, \Psi_{k, i}\right\rangle \psi_{k, i} \\
= & e^{-r\left(T-t_{s}\right)} \mathbf{E}\left(X \mid \sigma\left(\mathcal{R}_{j_{s}}\right)\right) \text { a.e. on } A_{k_{0}, i_{0}} .
\end{aligned}
$$

The assumption $V_{t_{s}}\left(\Psi_{k, i}\right)=0$, needed in Theorem 4.6, will not hold for a general $\mathrm{H}$ system. The following proposition provides a sufficient condition $V_{t_{s}}\left(\Psi_{k, i}\right)=0$ to be valid, a useful form of the result appears in Corollary 4.8.

Proposition 4.7. Consider an $H$-system where the associated Haar obligations $\Psi_{k, i}$ satisfy $\psi_{k, i}=$ $a \mathbf{1}_{A}+b \mathbf{1}_{B}$ with $A=E \cap F_{0}, B=E \cap F_{1}, E \in \mathcal{F}_{t_{s}}, F_{i} \in \mathcal{F}_{t_{s+1}}, i=0,1$ and

$$
a \mathbf{E}\left(\mathbf{1}_{F_{0}} \mid \mathcal{F}_{t_{s}}\right)+b \mathrm{E}\left(\mathbf{1}_{F_{1}} \mid \mathcal{F}_{t_{s}}\right)=0
$$

Then, $V_{t_{s}}\left(\Psi_{k, i}\right)=0$.

Proof. Notice that $\mathbf{E}\left(\psi_{k, i} \mid \mathcal{F}_{t_{s}}\right)=a \mathbf{1}_{E} \mathbf{E}\left(\mathbf{1}_{F_{0}} \mid \mathcal{F}_{t_{s}}\right)+b \mathbf{1}_{E} \mathbf{E}\left(1_{F_{1}} \mid \mathcal{F}_{t_{s}}\right)=0$.

Corollary 4.8. Consider the same setup as in Proposition 4.7 but also $E=A \cup B, E \in \mathcal{F}_{t_{s}}$, and instead of (4.7) assume the events $F_{i} \in \mathcal{F}_{t_{s+1}}$ to be independent with $\mathcal{F}_{t_{s}}$. Then, $V_{t_{s}}\left(\Psi_{k, i}\right)=0$.

Proof. We have

$$
0=\mathbf{E}\left(\psi_{k, i} \mid F_{t_{s}}^{n}\right)=a \mathbf{1}_{E} P\left(F_{0}\right)+b \mathbf{1}_{E} P\left(F_{1}\right)
$$

This implies (4.7). 
Remark 4.9. Corollary 4.8 applies when the atoms from $H$-systems are constructed by splitting a given event by means of an independent random variable. In particular it can be applied to processes with independent increments as in the Black and Scholes model in Section 6.

If the condition $V_{t_{s}}\left(\Psi_{k, i}\right)=0$ does not hold, we need to absorb the non self-financing nature of the portfolio as an additional approximating error. We do not analyze this error here but only provide the following informal argument.

Consider a re-balancing time $t_{1}$ and a Haar obligation $\Psi$ (starting at time $t_{1}$ and maturing at time $\left.t_{2}\right)$ such that $V_{t_{1}}(\Psi) \neq 0$. Given $w \in \Omega$ assume that there exists $A \in \mathcal{F}_{t_{1}}$ with $P(A)>0$ and $w \in A$ such that $\mathrm{E}_{A}(\Psi)=0$. Then, the exact error (including the sign but excluding interest rates for simplicity) is given by

$$
\mathrm{E}\left(X \mid F_{t_{1}}^{n}\right)-\frac{1}{P(A)} \int_{A} X d P
$$

We can then restart our approximations at $t_{1}$ with a higher resolution $n_{1}$ such that $A \in \mathcal{F}_{t_{1}}^{n_{1}}$, so that $\mathbf{E}\left(X \mid \mathcal{F}_{t_{1}}^{n_{1}}\right)=(1 / P(A)) \int_{A} X d P$. In this way we accumulate the non-self-financing nature of the portfolio as errors in the following way (define $n_{0} \equiv n$ ):

$$
\sum_{i=0}^{N-2} \mathrm{E}\left(X \mid \mathscr{F}_{t_{i+1}}^{n_{i}}\right)-\mathbf{E}\left(X \mid \mathscr{F}_{t_{i+1}}^{n_{i+1}}\right)
$$

A simple case where the errors (4.10) can be controlled a priori (i.e., at the start of hedging at $\left.t_{0}\right)$ is when hedging a derivative $X$ and there exist an integer $K$ and times $r_{0}, \ldots, r_{K}$ such that $X=X\left(S_{r_{0}}, \ldots, S_{r_{K}}\right)$.

\section{Portfolio of Characteristic Functions}

This section shows how to construct a self-financing portfolio $B \Pi_{\mathcal{R}}(X)_{t}$ to hedge $X$. The portfolio $B \Pi_{\mathcal{R}}(X)_{t}$ will also be re-balanced at times $t_{0}, \ldots, t_{N-1}$, replicating $e^{-r\left(T-t_{s}\right)} \mathbf{E}(X \mid$ $\left.\sigma\left(\mathcal{R}_{j_{s}}\right)\right)$ for $s=1, \ldots, N$. Taking into account Remark 4.5, we will dispense with writing the analogue of Theorem 4.6 for $B \Pi_{\mathcal{R}}(X)_{t}$, we mention that the sufficient conditions of Proposition 4.7 and Corollary 4.8 apply to this new portfolio as well.

We formalize $B \Pi_{\mathcal{R}}(X)_{t}$ as a vector-valued process that is constant on the intervals $t_{s-1} \leq t<t_{s}$. At time $t_{0}$ it is defined, for $t \in\left[t_{0}, t_{1}\right)$, by specifying its coordinates, namely how much to invest in each of the binary options,

$$
\begin{gathered}
e^{-r\left(T-t_{0}\right)} \mathbf{E}(X) \quad \text { invested in the bond and } \\
e^{-r\left(T-t_{0}\right)}\left(x_{k}[i] \mathbf{B}_{k, i}-\mathbf{E}(X)\right) \quad \text { where }(k, i) \in K_{j_{1}} .
\end{gathered}
$$

The cost of purchasing this portfolio is $V_{t_{0}}\left(B \Pi_{\mathcal{R}}(X)\right)=e^{-r\left(T-t_{0}\right)} \mathbf{E}(X)=e^{-r\left(T-t_{0}\right)} x_{0}[0]$. The inductive step will be to rebalance the portfolio at time $t_{s-1}$. Assume that at this time the 
price process is in the event $A_{k_{0}, i_{0}}$ with $\left(k_{0}, i_{0}\right) \in K_{j_{s-1}}$, and the value of this portfolio is $e^{-r\left(T-t_{s-1}\right)} x_{k_{0}}\left[i_{0}\right]$. We need to specify the coordinates of $B \Pi_{\mathcal{R}}(X)_{t}$, for $t_{s-1} \leq t<t_{s}$, namely,

$$
\begin{gathered}
e^{-r\left(T-t_{s-1}\right)} x_{k_{0}}\left[i_{0}\right] \quad \text { invested in the bond and } \\
e^{-r\left(T-t_{s-1}\right)}\left(x_{k}[i]-x_{k_{0}}\left[i_{0}\right]\right) \mathbf{B}_{k, i} \quad \text { where }(k, i) \in K_{j_{s}}^{k_{0}, i_{0}},
\end{gathered}
$$

and $K_{j_{s}}^{k_{0}, i_{0}} \equiv\left\{(k, i) \in K_{j_{s}}: A_{k, i} \subseteq A_{k_{0}, i_{0}}\right\}$.

$K_{j_{s}}^{k_{0}, i_{0}}$ reduces to $\left(k_{0}, i_{0}\right)$ if $A_{k_{0}, i_{0}}$ does not split in $p_{t_{s}}^{n}$.

In an analogous way to the done for $H \Pi_{\mathcal{R}}(X)$ it is possible to prove that the strategy $B \Pi_{\mathcal{R}}(X)$ is self-financing and replicates $e^{-r\left(T-t_{s}\right)} \mathbf{E}\left(X \mid \sigma\left(\mathcal{R}_{j_{s}}\right)\right)$ at $s=1, \ldots, N$. It should be clear that the hedging strategies $B \Pi_{\mathcal{R}}(X)$ and $H \Pi_{\mathcal{R}}(X)$ can be intermixed at different time intervals $\left[t_{s-1}, t_{s}\right)$.

\subsection{Compressed Approximations}

Let $u_{k_{i}} i \geq 0$ be a new indexing for our Haar system $\left\{u_{k}\right\}_{k \geq 0}$ that satisfies $\left|\left\langle X, u_{k_{i+1}}\right\rangle\right| \leq \mid$ $\left\langle X, u_{k_{i}}\right\rangle \mid$, except for $u_{k_{0}} \equiv u_{0}$. Define the $R$-term compressed approximation, which we will denote by $X_{R}^{c}$, by

$$
X_{R}^{c} \equiv \sum_{i=0}^{R}\left\langle X, u_{k_{i}}\right\rangle u_{k_{i}}
$$

As we will see, $X_{R}^{c}$ provides a very good approximation with a small volume of transactions and using small values of $R$. This implies that few binary options are required to implement the portfolio $Н П$. Notice that we have defined compression through the Haar representation, the same resulting approximating random variable (namely $X_{R}^{c}$ ) will also be used when computing volume of transactions for the portfolio $B \Pi$. This implies that the number of binary options required to implement the portfolio $B \Pi$ is about half the number of binary options required to implement the portfolio $Н П$. In practice we will apply the abovedescribed compression at the beginning of each time interval $\left[t_{s-1}, t_{s}\right)$ to the martingale differences as we explain next.

For fixed $n$ and a given sequence of times $t_{s}^{n}, s=0, \ldots, N_{n}$, let $\mathcal{F}_{t_{s}}^{n}$ represent the discrete skeleton atomic sigma algebra approximating that $\mathcal{F}_{t_{s}}$ (see Definition 3.2). We also assume, $t_{0}^{n}=t_{0}$ is the initial time, $\mathscr{F}_{t_{0}}$ the trivial sigma algebra and $t_{N_{n}}=T$-the final time. For simplicity, we will not display the dependency on $n$ in some of the notation, in particular we will write $N=N_{n}$ and $t_{s}=t_{s}^{n}$ (we will keep using $n$ to maintain the fundamental distinction between $\mathcal{F}_{t}^{n}$ and $\left.\mathcal{F}_{t}\right)$. Notice that by continuity of $S_{t}$ we can uniformly approximate $\mathbf{E}\left(\cdot \mid \mathcal{F}_{t}\right)(w)$ with $\mathbf{E}\left(\cdot \mid \boldsymbol{F}_{t}^{n}\right)(w)$ for $n$ sufficiently large.

For the sake of clarity we will add a superindex $t_{s}$ to the notation introduced in Section 2.1, to indicate, for example, that $\left\{A_{k, i}^{t_{s}}\right\},(k, i) \in K_{j_{s}}$ denotes the collection $p_{t_{s}}^{n}=\mathcal{R}_{j_{s}}$ of atoms generating $\mathcal{F}_{t_{\mathrm{s}}}^{n}$. We also add the super-index to other objects related to time $t_{s}$. Recall that $\left\{\mathcal{R}_{j}\right\}_{j \geq 0}$ is the binary sequence of partitions associated with the skeleton. Notice that $A_{k, i}^{t_{0}}=A_{0,0}^{t_{0}}=\Omega$. 
Define

$$
D_{t_{s-1}, t_{s}} \equiv \mathrm{E}\left(X \mid \mathcal{F}_{t_{s}}^{n}\right)-\mathbf{E}\left(X \mid \mathcal{F}_{t_{s-1}}^{n}\right), \quad s=1, \ldots, N
$$

Consider $\psi_{k, i} \in \mathcal{F}_{t_{s}}^{n}$, and then:

$$
\left\langle D_{t_{s-1}, t_{s}}, \psi_{k, i}\right\rangle \equiv\left\langle\mathrm{E}\left(X \mid \mathcal{F}_{t_{s}}^{n}\right)-\mathrm{E}\left(X \mid \mathcal{F}_{t_{s-1}}^{n}\right), \psi_{k, i}\right\rangle=\left\langle\mathrm{E}\left(X \mid \mathcal{F}_{t_{s}}^{n}\right), \psi_{k, i}\right\rangle=\left\langle X, \psi_{k, i}\right\rangle
$$

where the last two equalities follow from basic properties of the conditional expectation.

Noticing that the disjoint atoms $A_{j, l}^{t_{s-1}},(j, l) \in \mathcal{R}_{j_{s-1}}$ are a partition of $\Omega$ and using (3.6), we obtain the decomposition

$$
\begin{aligned}
D_{t_{s-1}, t_{s}} & =\sum_{(j, l) \in K_{j_{s-1}}} \mathbf{1}_{A_{j, l}^{t_{s-1}}} \sum_{m=j_{s-1}}^{j_{s}-1} \sum_{\left\{(k, i) \in H_{m}: A_{k, i} \subset A_{j, l}^{t_{s-1}}\right\}}\left\langle D_{t_{s-1}, t_{s}}, \psi_{k, i}\right\rangle \psi_{k, i} \\
& =\sum_{(j, l) \in K_{j_{s-1}}} \mathbf{1}_{A_{j, 1}^{t_{s-1}}} \sum_{m=j_{s-1}}^{j_{s-1}-1} \sum_{\left\{(k, i) \in H_{m}: A_{k, i} \subset A_{j, l}^{t_{s-1}}\right\}}\left\langle X, \psi_{k, i}\right\rangle \psi_{k, i} .
\end{aligned}
$$

Of course, $\psi_{k, i}=a_{k, i} \mathbf{1}_{A_{k+1,2 i}}+b_{k, i} \mathbf{1}_{A_{k+1,2 i+1}}$ is the Haar function at node $A_{k, i}$ (see (2.5)). In applications (see Section 6) we will perform compression by ordering the Fourier coefficients appearing in (4.17). This results in a compressed martingale difference $D_{t_{s-1}, t_{s}}^{c}$, it is the same function that is also expanded in the characteristic functions basis.

Recalling the orthonormal system

$$
\phi_{k, i}^{t_{s}} \equiv \frac{1}{\sqrt{P\left(A_{k, i}^{t_{s}}\right)}} \mathbf{1}_{A_{k, i}^{t_{s}}}, \quad \text { for }(k, i) \in K_{j_{s}}
$$

we can then write, for $w \in A_{j, l}^{t_{s-1}}$,

$$
D_{t_{s-1}, t_{s}}^{c}(w)=\sum_{\left\{(k, i) \in K_{j s}: A_{k, i}^{t_{s}} \subset A_{j, l}^{t_{s-1}}\right\}}\left\langle D_{t_{s-1}, t_{s}}^{c}, \phi_{k, i}^{t_{s}}\right\rangle \phi_{k, i}^{t_{s}}(w) .
$$

To sum up, in practice both portfolios $B \Pi$ and $H \Pi$, at each interval $\left[t_{s-1}, t_{s}\right)$, implement $D_{t_{s-1}, t_{s}}^{c}$ and hence provide the same approximation. Their implementation in terms of binary options is different and, hence, the resulting volume of transactions differ. This last topic is studied in Section 5.

\section{Volume of Transactions}

The results in the present section provide upper bounds, in the mean and pointwise, for the volume of transactions required to implement the portfolios ВП and $Н П$ (introduced 
in Section 4). Proposition 5.13 proves that the volume of transactions for the portfolio $В П$ is smaller the one for $H \Pi$; because of this result, a justification for our reliance on the portfolio $H \Pi$ is in order. It is only through the use of $Н \Pi$ that we can perform compression as described in Section 4.2; in particular, this allows to use few binary options while retaining a small error of approximation. As indicated above, the compressed approximation is also the one to be implemented by $B \Pi$. Moreover, the upper bounds for the volume of transactions rely on an analysis of the behavior of the inner products defining $Н П$. Other aspects of our approximations used to obtain the upper bounds are the following: they approximate the relevant conditional expectations, the martingale property is used in the self-financing nature of the portfolios, the orthogonality of the martingale differences holds, and the localized property of the basis functions is used throughout (as in (5.10)).

We believe that the quality of the upper bounds described in the present section provides evidence of the efficiency of our approximations as far as volume of transactions is concerned. We have written the results for the portfolios $B \Pi$ and $H \Pi$ but they remain valid for their compressed versions as well. Numerical results are presented in Section 6.

The results assume the existence of a skeleton approximation (and, hence, continuity of $S_{t}$ will be assumed) as described in Section 3; this assumption is to make sure we have available a family of finite $H$-systems that approximate the given process.

Definition 5.1. Consider the binary options $\mathbf{B}_{k, i}^{t_{s}}=\left(\mathbf{1}_{A_{k, i}^{t_{s}}}(t) \equiv \mathbf{1}_{\left[t_{s-1}, T\right]}(t) \mathbf{1}_{A_{k, i}^{t_{s}}}\right),(k, i) \in K_{j_{s}}$ as described in (4.1). The volume of transactions, at time $t_{s-1}$, required for a financial implementation of such an option is denoted by $\mathrm{VT}_{t_{s-1}}\left(\mathbf{B}_{k, i}^{t_{s}}\right)$ and equals its value, that is,

$$
\mathrm{VT}_{t_{s-1}}\left(\mathbf{B}_{k, i}^{t_{s}}\right)=e^{-r\left(t_{s}-t_{s-1}\right)} \mathbf{E}\left(\mathbf{1}_{A_{k, i}^{t_{s}}} \mid \mathcal{F}_{t_{s-1}}\right)
$$

Definition 5.2. The volume of transactions necessary to implement a portfolio $\Pi$ represented, in terms of the above binary options, by $\Pi=\sum c_{k, i} \mathbf{B}_{k, i}^{t_{s}}$, is given by

$$
\mathrm{VT}_{t_{s-1}}(\Pi) \equiv \sum\left|c_{k, i}\right| e^{-r\left(t_{s}-t_{s-1}\right)} \mathbf{E}\left(\mathbf{1}_{A_{k, i}^{t_{s}}} \mid \mathcal{F}_{t_{s-1}}\right)
$$

Remark 5.3. The binary options appearing in the representation of the random variable $\Pi$ are not assumed to constitute a fixed linearly independent set; therefore $\Pi$ could be represented in different ways in terms of the linearly dependent set of characteristic functions $\mathbf{1}_{A_{k, i}^{t_{s}}}$. This implies, in particular, that the volume of transactions as defined in (5.2) depends on the specific representation of $\Pi$ in terms of binary options.

Lemma 5.4. Given the binary option $\mathbf{B}_{k, i}^{t_{s}}=\mathbf{1}_{A_{k, i}^{t_{s}}}$, with $A_{k, i}^{t_{s}} \in \mathcal{F}_{t_{s}}^{n}$ and $A_{k, i}^{t_{s}} \subseteq A_{j, l}^{t_{s-1}} \in \mathcal{F}_{t_{s-1}}^{n}$ for $(k, i) \in K_{j_{s}}$, then

$$
\mathbf{E}\left(\mathbf{1}_{A_{k, i}^{t_{s}}} \mid \mathcal{F}_{t_{s-1}}^{n}\right)=\frac{P\left(A_{k, i}^{t_{s}}\right)}{P\left(A_{j, l}^{t_{s-1}}\right)} \mathbf{1}_{A_{j, l}^{t_{s-1}}}
$$


and so

$$
\mathbf{E}\left(V T_{t_{s-1}}\left(\mathbf{B}_{k, i}^{t_{s}}\right)-e^{-r\left(t_{s}-t_{s-1}\right)} \mathbf{E}\left(\mathbf{1}_{A_{k, i}^{t_{s}}} \mid \mathcal{F}_{t_{s-1}}^{n}\right)\right)=0
$$

Proof. The proof follows by noticing that $A_{j, l}^{t_{s-1}}$ is an atom of the atomic sigma algebra $\mathbb{F}_{t_{s-1}}^{n}$.

In order to obtain pointwise upper bounds we will need to substitute $\mathbf{E}\left(\mathbf{1}_{A_{k, i}^{t_{s}}} \mid \mathcal{F}_{t_{s-1}}\right)$ by $\mathbf{E}\left(\mathbf{1}_{A_{k, i}^{t_{s}}} \mid \mathcal{F}_{t_{s-1}}^{n}\right)$ in (5.1) (and hence also in (5.2)). The resulting expressions will be called approximate values and denoted by $\widehat{\mathrm{VT}}_{t_{s-1}}\left(\mathbf{B}_{k, i}^{t_{s}}\right)$. By means of the same substitution, one can also obtain the approximate values $\widehat{\mathrm{VT}}_{t_{s-1}}(\Pi)$ and $\widehat{\mathrm{VT}}_{t_{s-1}}\left(D_{t_{s-1}, t_{s}}\right)$ using (5.1) and (5.6), respectively. Notice that the approximate values converge pointwise to the corresponding exact values as $n \rightarrow \infty$. Therefore, we can concentrate on establishing pointwise error bounds for the approximated quantities.

Remark 5.5. Under the conditions of Corollary 4.8, and in particular under the setting of Section 6, we have $\mathrm{VT}_{t_{s-1}}\left(D_{t_{s-1}, t_{s}}\right)=\widehat{\mathrm{VT}}_{t_{s-1}}\left(D_{t_{s-1}, t_{s}}\right)$ and hence also $\mathrm{VT}_{t_{s-1}}(\Pi)=\widehat{\mathrm{VT}}_{t_{s-1}}(\Pi)$. Equation (5.4) will be used in several instances to justify the equality $\mathrm{E}\left(\operatorname{VT}\left(D_{t_{s-1}, t_{s}}\right)\right)=$ $\mathbf{E}\left(\widehat{\mathrm{VT}}\left(D_{t_{s-1}, t_{s}}\right)\right)$, from which $\mathbf{E}\left(\mathrm{VT}_{t_{0}}(\Pi)\right)=\mathbf{E}\left(\widehat{\mathrm{VT}}_{t_{0}}(\Pi)\right)$ follows (these expectation results follow in general, that is independently of the validity of Corollary 4.8).

We will analyze the volume of transactions needed to implement the binary portfolio $(B \Pi)$ and the Haar portfolio $(H \Pi)$ introduced in Section 4.1. The notation will be designed, as much as possible, so the developments cover both cases simultaneously. When stating results that apply to both portfolios we will designate either of them by $\Pi$ :

Lemma 5.6. The following expression provides the volume of transactions required for a financial implementation of the portfolio(s) П.

$$
V T_{t_{0}}(\Pi)=\sum_{s=1}^{N} \sum_{(k, i) \in K_{j_{s-1}}} \mathbf{1}_{A_{k, i}^{t_{s-1}}} V T_{t_{s-1}}\left(D_{t_{s-1}, t_{s}}\right) .
$$

Proof. It is enough to establish (5.5) for any of the two portfolios. we consider $Н П$ for definiteness and argue by induction. Recall that $A_{0,0}^{0}=\Omega$, given that $e^{-r\left(T-t_{0}\right)} \mathrm{E}(X)$ is invested in the bond; at time $t_{1}$ the Haar portfolio provides the approximation $\mathrm{E}\left(X \mid \mathcal{F}_{t_{1}}^{n}\right)$. Therefore, only the difference $D_{t_{0}, t_{1}}$ require a financial realization using binary options; the associated volume of transactions is given by $\mathrm{VT}_{t_{0}}\left(D_{t_{0}, t_{1}}\right)$. Reasoning inductively, at time $t_{s-1}$, the value of the Haar hedging strategy is $\mathrm{E}\left(X \mid \mathcal{F}_{t_{s-1}}^{n}\right)$. Therefore, in order to achieve the approximation $\mathrm{E}\left(X \mid \mathcal{F}_{t_{s}}^{n}\right)$ at time $t_{s}$, only the difference $D_{t_{s-1}, t_{s}}$ requires a financial realization using binary options; the associated volume of transactions is given by $\operatorname{VT}_{t_{s-1}}\left(D_{t_{s-1}, t_{s}}\right)(w)$ where $w$ belongs to some $A_{k, i}^{t_{s-1}}$ with $(k, i) \in K_{j_{s-1}}$. This completes the inductive argument and gives (5.5). An analogous reasoning provides the argument for the case of the $B \Pi$ portfolio.

\subsection{Representation by Characteristic Functions}

From (4.18), (4.19), and (5.2), we obtain the following proposition. 
Proposition 5.7. For $w \in A_{j, l}^{t_{s-1}}$

$$
\begin{gathered}
V T_{t_{s-1}}\left(D_{t_{s-1}, t_{s}}\right)(w)=e^{-r\left(t_{s}-t_{s-1}\right)} \sum_{\left\{(k, i) \in K_{j s}: A_{k, i}^{t_{s}} \subseteq A_{j, l}^{t_{s-1}}\right\}} \frac{\left\langle D_{t_{s-1}, t_{s}}, \phi_{k, i}^{t_{s}}\right\rangle}{\sqrt{P\left(A_{k, i}^{t_{s}}\right)}} \mathbf{E}\left(\mathbf{1}_{A_{k, i}^{t_{s}}} \mid \boldsymbol{F}_{t_{s-1}}\right)(w) . \\
\widehat{V T}_{t_{s-1}}\left(D_{t_{s-1}, t_{s}}\right)(w)=e^{-r\left(t_{s}-t_{s-1}\right)} \frac{\mathbf{1}_{A_{j, 1}^{t_{s-1}}}}{P\left(A_{j, l}^{t_{s-1}}\right)}\left\|\mathbf{1}_{A_{j, l}^{t_{s-1}}} D_{t_{s-1}, t_{s}}\right\|_{1}
\end{gathered}
$$

Corollary 5.8. Consider an arbitrary path $\left\{A_{k_{s}, i_{s}}^{t_{s}}\right\}_{s=1, \ldots, N^{\prime}}$ that is, $A_{k_{s}, i_{s}}^{t_{s}} \subseteq A_{k_{s-1}, i_{s-1}}^{t_{s-1}}$. Then,

$$
\widehat{V T}_{t_{0}}(B \Pi)=\sum_{s=1}^{N} e^{-r\left(t_{s}-t_{s-1}\right)} \frac{1}{P\left(A_{k_{s}, i_{s}}^{t_{s}}\right)}\left\|\mathbf{1}_{A_{k_{s}, i_{s}}^{t_{s}}} D_{t_{s-1}, t_{s}}\right\|_{1^{\prime}} \text { a.e. on } \cap A_{k_{s}, i_{s}}^{t_{s}} \text {. }
$$

\section{Corollary 5.9.}

$$
\mathbf{E}\left(V T_{t_{0}}(B \Pi)\right)=\sum_{s=1}^{N} e^{-r\left(t_{s}-t_{s-1}\right)}\left\|D_{t_{s-1}, t_{s}}\right\|_{1} \leq \sum_{s=1}^{N} e^{r\left(t_{s}-t_{s-1}\right)}\left\|D_{t_{s-1}, t_{s}}\right\|_{2}
$$

Proof. Notice that

$$
\bigcup_{\left\{(k, i) \in K_{j s}: A_{k, i}^{t_{s}} \in A_{j, l}^{t_{s-1}}\right\}} A_{k, i}^{t_{s}}=A_{j, l}^{t_{s-1}}, \quad \bigcup_{(j, l) \in K_{j_{s-1}}} A_{j, l}^{t_{s-1}}=\Omega,
$$

and also, $A_{k, i}^{t_{s}} \cap A_{k^{\prime}, i^{\prime}}^{t_{s}}=\emptyset$. Using (5.5), (5.6), and $A_{j, l}^{t_{s-1}} \in \mathcal{F}_{t_{s-1}}$, we obtain

$$
\begin{gathered}
\mathbf{E}\left(\mathrm{VT}_{t_{0}}(B \Pi)\right)=\sum_{s=1}^{N} e^{-r\left(t_{s}-t_{s-1}\right)} \sum_{(j, l) \in K_{j_{s-1}}} \sum_{\left\{(k, i) \in K_{j s}: A_{k, i}^{t_{s}} \subseteq A_{j, l}^{t_{s-1}}\right\}} \frac{\left|\left\langle D_{t_{s-1}, t_{s}}, \mathbf{1}_{A_{k, i}^{t_{s}}}\right\rangle\right|}{P\left(A_{k, i}^{t_{s}}\right)} \mathbf{E}\left(\mathbf{1}_{A_{j, l}^{t_{s-1}}} \mathbf{E}\left(\mathbf{1}_{A_{k, i}^{t_{s}}} \mid \boldsymbol{F}_{t_{s-1}}\right)\right) \\
=\sum_{s=1}^{N} e^{-r\left(t_{s}-t_{s-1}\right)}\left\|D_{t_{s-1}, t_{s}}\right\|_{1} .
\end{gathered}
$$

The inequality in (5.9) follows from Cauchy-Schwarz.

\subsection{Representation by Haar Functions}

The previous bounds for the volume of transactions did not use the Haar coefficients. In this section we deal with the Haar portfolio $H \Pi$, this amounts to representing the martingale differences using the Haar functions instead of the characteristic functions. 
Remark 5.10. Recalling the tree notation introduced in Section 2.1, we may set $\tau_{A_{j, l}^{t_{s-1}}}$ to denote the tree containing its root $A_{j, l}^{t_{s-1}}$ and internal nodes from $\cup_{m=j_{s-1}}^{j_{s}-1}\left\{A_{k, i} \in H_{m}\right\}$. So the double

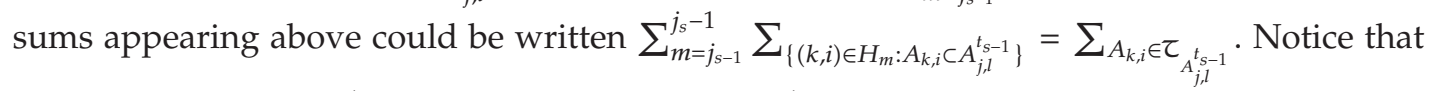
the condition $A_{k, i} \subset A_{j, l}^{t_{s-1}}$ includes the case $A_{k, i}=A_{j, l}^{t_{s-1}}$.

Using (5.2), the volume of transactions associated with decomposition (4.17) is given in the following Lemma.

Lemma 5.11. For $w \in A_{j, l}^{t_{s-1}}$,

$$
\begin{aligned}
V T_{t_{s-1}}^{H}\left(D_{t_{s-1}, t_{s}}\right)(w)= & e^{-r\left(t_{s}-t_{s-1}\right)} \sum_{m=j_{s-1}}^{j_{s}-1} \sum_{\left\{(k, i) \in H_{m}: A_{k, i} \subset A_{j, l}^{t_{s-1}}\right\}}\left|X, \psi_{k, i}\right| \\
& \times\left(\left|a_{k, i}\right| \mathbf{E}\left(\mathbf{1}_{A_{k+1,2 i}} \mid \mathcal{F}_{t_{s-1}}\right)(w)+\left|b_{k, i}\right| \mathbf{E}\left(\mathbf{1}_{A_{k+1,2 i+1}} \mid \mathcal{F}_{t_{s-1}}\right)(w)\right) .
\end{aligned}
$$

As previously indicated, the volume of transactions depends on the representation and we have used the notation $V T^{H}$ to highlight this fact. The approximate version of (5.12) is $\widehat{V} T_{t_{s-1}}^{H}\left(D_{t_{s-1}, t_{s}}\right)$ and is obtained by replacing $\mathcal{F}_{t_{s-1}}$ by $\mathcal{F}_{t_{s-1}}^{n}$ in (5.12).

Lemma 5.12. For $w \in A_{j, l}^{t_{s-1}}$,

$$
\begin{aligned}
& \widehat{V T}_{t_{s-1}}^{H}\left(D_{t_{s-1}, t_{s}}\right)(w)= \\
& e^{-r\left(t_{s}-t_{s-1}\right)} \frac{\mathbf{1}_{A_{j, 1}^{t_{s-1}}}}{P\left(A_{j, l}^{t_{s-1}}\right)} \sum_{m=j_{s-1}}^{j_{s}-1} \sum_{\left\{(k, i) \in H_{m}: A_{k, i} \subset A_{j, l}^{t_{s-1}}\right\}}\left|\left\langle X, \psi_{k, i}\right\rangle\right|\left\|\psi_{k, i}\right\|_{1} .
\end{aligned}
$$

The following proposition tells us that the portfolio $В \Pi$ has a smaller volume of transactions than the portfolio $Н \Pi$. Whenever appropriate we will use $\mathrm{VT}^{B}$ to denote the volume of transactions for the portfolio $В П$.

Proposition 5.13. One has,

$$
\widehat{V T}_{t_{s-1}}^{B}\left(D_{t_{s-1}, t_{s}}\right) \leq \widehat{V} T_{t_{s-1}}^{H}\left(D_{t_{s-1}, t_{s}}\right)
$$

Therefore,

$$
\widehat{V T}_{t_{0}}(B \Pi) \leq \widehat{V} T_{t_{0}}(H \Pi)
$$

Remark 5.14. Of course, Remark 5.5 applies and the above proposition implies the corresponding results after taking expectations. Moreover, Proposition 5.13 also holds if $D_{t_{s-1}, t_{s}}$ is replaced for any random variable $Y \in \mathcal{F}_{t_{s}}^{n}$, in particular $Y=D_{t_{s-1}, t_{s}}^{c}$, namely the compressed version of $D_{t_{s-1}, t_{s}}$. 
Proof. Making use of (4.16) and the triangle inequality,

$$
\frac{\mathbf{1}_{A_{j, l}^{t_{s-1}}}}{P\left(A_{j, l}^{t_{s-1}}\right)}\left\|\mathbf{1}_{A_{j, l}^{t_{s-1}}} D_{t_{s-1}, t_{s}}\right\|_{1} \leq \frac{\mathbf{1}_{A_{j, l}^{t_{s-1}}}}{P\left(A_{j, l}^{t_{s-1}}\right)} \sum_{m=j_{s-1}}^{j_{s}-1} \sum_{\left\{(k, i) \in H_{m}: A_{k, i} \subset A_{j, l}^{t_{s-1}}\right\}}\left|\left\langle X, \psi \psi_{k, i}\right\rangle\right|\left\|\psi_{k, i}\right\|_{1} .
$$

Then, (5.14) follows after observing (5.7) and (5.12).

In the computations that follow we assume that our approximating martingale is regular [19], namely for all parents nodes $A_{k, i}$ and its children we have,

$$
P\left(A_{k+1, l}\right) \leq \delta P\left(A_{k, i}\right), \quad l=2 i, 2 i+1,
$$

where $\delta \geq 1 / 2$ is the same for all nodes. For simplicity we will take $\delta=1 / 2$ as other choices will make the statements of our results more complex. Also, by means of Definition 5.15, nodes $A_{k, i}$ belonging to a tree $\tau_{n}$ are classified according to their size relative to the inner products $\left|\left\langle X, \psi_{k, i}\right\rangle\right|$.

Definition 5.15. Consider a node $A_{k, i}$ belonging to a tree $\tau_{n}$ associated with a given $H$-system satisfying $\left\langle X, \psi_{k, i}\right\rangle \neq 0$. The node $A_{k, i}$ is said to be of type I if

$$
\left|\left\langle X, \psi_{k, i}\right\rangle\right|<\sqrt{P\left(A_{k, i}\right)}
$$

Furthermore, it is said to be of type II if $\left|\left\langle X, \psi_{k, i}\right\rangle\right| \geq \sqrt{P\left(A_{k, i}\right)}$.

Also define $\epsilon_{k, i}$ by: $\left|\left\langle X, \psi_{k, i}\right\rangle\right|=P\left(A_{k, i}\right)^{1 / 2+\epsilon_{k, i}}$. Notice that $\epsilon_{k, i}>0$ whenever $A_{k, i}$ is of type I and that $\epsilon_{k, i} \leq 0$ whenever $A_{k, i}$ is of type II.

For the following lemma, consider a generic node $A_{j, l}^{t_{s-1}}$ of a given $H$-system and its associated tree $\tau_{A_{j, l}^{t_{s-1}}}$. To simplify the notation, nodes $A_{k, i}$ will always satisfy $A_{k, i} \in \tau_{A_{j, l}^{t_{s-1}}}$. In Lemma 5.16 we will make use of the following definition

$$
\begin{gathered}
\epsilon_{m} \equiv \min \left\{\epsilon_{k, i}:(k, i) \in H_{m}, A_{k, i} \text { of type I }\right\} \\
\epsilon_{t_{s-1}} \equiv \min \left\{\epsilon_{m}: j_{s-1}<m \leq j_{s}\right\} .
\end{gathered}
$$

Notice that $\epsilon_{t_{s-1}}>0$ and its value depend on the given node $A_{j, l}^{t_{s-1}}$. 
Lemma 5.16. Assume a given $H$-system is regular with $\delta=1 / 2$. Let $0 \leq q_{m}^{m}$ be the number of instances for which nodes at level $m$ of the tree $\tau_{A_{j, l}^{t_{s-1}}}$ are of type I and $r_{s} \equiv j_{s}-j_{s-1}$. Then,

$$
\begin{aligned}
\sum_{A_{k, i} \in \mathcal{C}_{A_{j, l}^{t_{s-1}}} \sqrt{P\left(A_{k, i}\right)}\left|\left\langle X, \psi_{k, i}\right\rangle\right|} & \leq\left(P\left(A_{j, l}^{t_{s-1}}\right)\right)^{1+\epsilon_{t_{s-1}}} \sum_{m=0}^{r_{s}}\left(q_{m} \delta^{1+\epsilon_{m}}\right)^{m}+\sum_{A_{k, i} \text { of type II }}\left|\left\langle X, \psi_{k, i}\right\rangle\right|^{2} \\
& \leq\left(P\left(A_{j, l}^{t_{s-1}}\right)\right)^{1+\epsilon_{t_{s-1}}} \frac{1}{1-2 \delta^{1+\epsilon_{t_{s-1}}}}+\left\|\mathbf{1}_{A_{j, l}^{t_{s-1}}} D_{t_{s-1}, t_{s}}\right\|_{2}^{2} \cdot
\end{aligned}
$$

Proof. Considering the fact that $\mathcal{R}_{m}$ can have at most $2^{m}$ members, hence $q_{m} \leq 2$, we obtain

$$
\begin{aligned}
\sum_{A_{k, i} \text { of type I }} \sqrt{P\left(A_{k, i}\right)}\left|\left\langle X, \psi_{k, i}\right\rangle\right| & \leq \sum_{m=0}^{r_{s}}\left(q_{m} \delta^{1+\epsilon_{m}}\right)^{m}\left(P\left(A_{j, l}^{t_{s-1}}\right)\right)^{1+\epsilon_{m}} \\
& \leq\left(P\left(A_{j, l}^{t_{s-1}}\right)\right)^{1+\epsilon_{t_{s-1}}} \sum_{m=0}^{r_{s}}\left(q_{m} \delta^{1+\epsilon_{m}}\right)^{m} \leq\left(P\left(A_{j, l}^{t_{s-1}}\right)\right)^{1+\epsilon_{t_{s-1}}} \frac{1}{1-2 \delta^{1+\epsilon_{t_{s-1}}}} .
\end{aligned}
$$

According to the decomposition,

$$
\begin{aligned}
\sum_{A_{k, i} \in \mathcal{T}_{A_{j, i}^{t}} \sqrt{j_{j, i}}} \sqrt{P\left(A_{k, i}\right)}\left|\left\langle X, \psi_{k, i}\right\rangle\right|= & \sum_{A_{k, i} \text { of type I }} \sqrt{P\left(A_{k, i}\right)}\left|\left\langle X, \psi_{k, i}\right\rangle\right| \\
& +\sum_{A_{k, i} \text { of type II }} \sqrt{P\left(A_{k, i}\right)}\left|\left\langle X, \psi_{k, i}\right\rangle\right|,
\end{aligned}
$$

In order to complete the proof, it only remains to bound the second summation in the righthand side of (5.22). As we remarked before, $\left\langle X, \psi_{k, i}\right\rangle=\left\langle D_{t_{s-1}, t_{s}}, \psi_{k, i}\right\rangle$, then

$$
\sum_{A_{k, i} \text { of type II }} \sqrt{P\left(A_{k, i}\right)}\left|\left\langle X, \psi_{k, i}\right\rangle\right| \leq \sum_{A_{k, i} \text { of type II }}\left|X, \psi_{k, i}\right|^{2} \leq\left\|\mathbf{1}_{A_{j, l}^{t_{s-1}}} D_{t_{s-1}, t_{s}}\right\|_{2}^{2} .
$$

From the above lemma we deduce the following result on static Hedging for the Haar portfolio.

Corollary 5.17. Assume the same hypothesis as in Lemma 5.16 and consider $V T_{t_{0}}(H \Pi)$ in (5.5) with $N=1$, that is, a static replication and so $A_{j, l}^{t_{0}}=A_{0,0}^{t_{0}}=\Omega$; then,

$$
\widehat{V T}_{t_{0}}(H \Pi)=e^{-r\left(T-t_{0}\right)} \sum_{A_{k, i} \in \mathcal{Z}_{\Omega}} \sqrt{P\left(A_{k, i}\right)}\left|\left\langle X, \psi_{k, i}\right\rangle\right|
$$




$$
\begin{aligned}
& \leq 2 \delta e^{-r\left(T-t_{0}\right)} \times\left(\sum_{m=0}^{r_{1}}\left(q_{m} \delta^{1+\epsilon_{m}}\right)^{m}+\sum_{A_{k, i} \text { of type II }}\left|\left\langle X, \psi_{k, i}\right\rangle\right|^{2}\right) \\
& \leq 2 \delta e^{-r\left(T-t_{0}\right)} \times\left(\frac{1}{1-2 \delta^{1+\epsilon_{t_{0}}}}+\left\|D_{t_{0}, T}\right\|_{2}^{2}\right) .
\end{aligned}
$$

Proof. Using (5.12) with $s=1$, the regularity assumption, and the constraints $\mathbf{E}\left(\psi_{k, i}\right)=0$ and $\mathrm{E}\left(\psi_{k, i}^{2}\right)=1$, we obtain

$$
\begin{aligned}
\widehat{\mathrm{VT}}_{t_{0}}(H \Pi)= & \mathrm{VT}_{t_{0}}^{H}\left(D_{0, T}\right) \leq 2 \delta e^{-r\left(T-t_{0}\right)} \\
& \times\left(\sum_{m=0\left\{(k, i) \in H_{m}\right\}}^{j_{1}-1} \sqrt{P\left(A_{k, i}\right)}\left|\left\langle X, \psi_{k, i}\right\rangle\right|\right) .
\end{aligned}
$$

The result (5.24) then follows from Lemma 5.16.

In Theorem 5.18 we will make use of the following definition: consider a given path $\left\{A_{j_{s}, l_{s}}^{t_{s}}\right\}_{s=0}^{N}$ and

$$
\epsilon \equiv \min _{s=0, \ldots, N-1} \epsilon_{t_{s}}
$$

Notice that $\epsilon>0$ and it depends on the given path.

The next theorem provides pointwise bounds for the volume of transactions for the portfolio $H \Pi$ in the general dynamic case (so $N \geq 1$ ). Corollary 5.17 is a special case of this theorem.

Theorem 5.18. Consider an arbitrary path $\left\{A_{j_{s}, l_{s}}^{t_{s}}\right\}_{s=0}^{N}$ with $\omega \in \cap A_{j_{s}, l_{s}}^{t_{s}}$ and the same hypothesis as in Lemma 5.16. At each node $A_{j_{s}, l_{s}}^{t_{s}}$ define the quantities $q_{t_{s-1}}=\sum_{m=0}^{r_{s}}\left(q_{m} \delta^{1+\epsilon_{m}}\right)^{m}$ where the $q_{m}$ are as in Lemma 5.16. Then,

$$
\begin{aligned}
\widehat{V T}_{t_{0}}(H \Pi)(w) & =\sum_{s=0}^{N-1} e^{-r\left(t_{s+1}-t_{s}\right)} \frac{1}{P\left(A_{j_{s}, l_{s}}^{t_{s}}\right)} \sum_{k, i \in \mathcal{C}_{A_{s, l s}^{t_{s}}} \sqrt{P\left(A_{k, i}\right)}\left|\left\langle X, \psi_{k, i}\right\rangle\right|} \\
& \leq 2 \delta \sum_{s=0}^{N-1} e^{-r\left(t_{s+1}-t_{s}\right)}\left[\left(P\left(A_{j_{s}, l_{s}}^{t_{s}}\right)\right)^{\epsilon_{t_{s}}} q_{t_{s}}+\frac{1}{P\left(A_{j_{s}, l_{s}}^{t_{s}}\right)} \sum_{A_{k, i} \text { of type II }}\left|\left\langle X, \psi_{k, i}\right\rangle\right|^{2}\right] \\
& \leq\left(\frac{1}{\left(1-2 \delta^{1+\epsilon}\right)} \frac{1}{\left(1-\delta^{e}\right)}+\sum_{s=0}^{N-1} \frac{1}{P\left(A_{j_{s}, l_{s}}^{t_{s}}\right)} \| 1_{\left.A_{j_{s}, l_{s}}^{t_{s}} D_{t_{s}, t_{s+1}} \|_{2}^{2}\right) .}\right.
\end{aligned}
$$


Proof. Using the approximated version of (5.12), the regularity assumption, and the constraints $\mathbf{E}\left(\psi_{k, i}\right)=0$ and $\mathbf{E}\left(\psi_{k, i}^{2}\right)=1$, if $\omega \in \cap A_{j_{s}, l_{s}}^{t_{s}}$, we obtain

$$
\begin{aligned}
\widehat{\mathrm{VT}}_{t_{s}}^{H}\left(D_{t_{s}, t_{s+1}}\right)(w) \leq & 2 \delta e^{-r\left(t_{s+1}-t_{s}\right)} \\
& \times \frac{1}{P\left(A_{j_{s}, l_{s}}^{t_{s}}\right)} \sum_{m=j_{s}}^{j_{s+1}-1} \sum_{\left\{(k, i) \in H_{m}: A_{k, i} \subset A_{j_{s, l s}}^{t_{s}}\right\}} \sqrt{P\left(A_{k, i}\right)}\left|\left\langle X, \psi_{k, i}\right\rangle\right| .
\end{aligned}
$$

Using the first inequality in (5.20), inequality (5.32), and (5.5), we obtain for $\omega \in \cap A_{j_{s}, l_{s}}^{t_{s}}$

$$
\begin{aligned}
\widehat{\mathrm{VT}}_{t_{0}}(H \Pi)(w) \leq & 2 \delta \sum_{s=0}^{N-1} e^{-r\left(t_{s+1}-t_{s}\right)} \\
& \times\left[\left(P\left(A_{j_{s}, l_{s}}^{t_{s}}\right)\right)^{\epsilon_{t_{s}}} q_{t_{s}}+\frac{1}{P\left(A_{j_{s}, l_{s}}^{t_{s}}\right)} \sum_{A_{k, i} \in \tau_{A_{s, l_{s}}^{t_{s}}} \text { of type II }}\left|\left\langle X, \psi_{k, i}\right\rangle\right|^{2}\right] .
\end{aligned}
$$

Notice that

$$
\sum_{s=0}^{N-1}\left(P\left(A_{j_{s}, l_{s}}^{t_{s}}\right)\right)^{\epsilon_{t_{s}}} \leq 1+\left(\delta^{j_{1}}\right)^{\epsilon_{t_{1}}}+\cdots+\left(\delta^{j_{N-1}}\right)^{\epsilon_{t_{N-1}}}, \quad q_{t_{s}} \leq \sum_{m=0}^{r_{s+1}}\left(q_{m} \delta^{1+\epsilon}\right)^{m} \leq \frac{1}{\left(1-2 \delta^{1+\epsilon}\right)} .
$$

Therefore,

$$
\widehat{\mathrm{VT}}_{t_{0}}(H \Pi)(w) \leq 2 \delta\left(\frac{1}{\left(1-2 \delta^{1+\epsilon}\right)} \frac{1}{\left(1-\delta^{\epsilon}\right)}+\sum_{s=0}^{N-1} \frac{1}{P\left(A_{j_{s}, l_{s}}^{t_{s}}\right)}\left\|\mathbf{1}_{A_{j, l}^{t_{s}}} D_{t_{s}, t_{s+1}}\right\|_{2}^{2}\right)
$$

Notice that Theorem 5.18 provides two inequalities, the term after the first inequality represents a fine bound and the term after the second inequality presents a coarser one. An analogous remark applies to Corollary 5.17. In the following Theorem we present an upper bound for the mean of the volume of transactions and make use only of the coarser versions of previous inequalities. The reason for this is that, at this point, we are mainly interested in showing how the upper bound for the mean value relates to the number of time steps $N$.

Theorem 5.19. Consider the same hypothesis as in Theorem 5.18 and assume that $\epsilon$ is constant over all the tree paths. Then,

$$
\mathbf{E}\left(V T_{t_{0}}(H \Pi)\right) \leq \frac{2}{\left(1-2 \delta^{1+e}\right)}+\left\|\mathbf{E}\left(X \mid \mathcal{F}_{T}^{n}\right)-\mathbf{E}(X)\right\|_{2}^{2} .
$$


Table 1: Relative errors, between $X$ and $X_{R}^{c}$, in terms of number of transactions and scales. Single European call. Values of parameters as in Figure 1.

\begin{tabular}{lcccccc}
\hline No. of transactions & $J=6$ & $J=8$ & $J=10$ & $J=12$ & $J=14$ & $J=16$ \\
\hline$R=5$ & 0.1907 & 0.1907 & 0.1907 & 0.1907 & 0.1907 & 0.1907 \\
$R=15$ & 0.0849 & 0.0631 & 0.0627 & 0.0627 & 0.0627 & 0.0627 \\
$R=30$ & 0.0769 & 0.0421 & 0.0323 & 0.0312 & 0.0312 & 0.0312 \\
$R=64$ & 0.0767 & 0.0361 & 0.02041 & 0.0157 & 0.0147 & 0.0147 \\
$R=128$ & NA & 0.0351 & 0.0173 & 0.0101 & 0.0079 & 0.0074 \\
$R=256$ & NA & 0.0351 & 0.0166 & 0.0084 & 0.0050 & 0.0040 \\
\hline
\end{tabular}

Proof. Combining (5.5) with (5.12), we obtain

$$
\mathbf{E}\left(\mathrm{VT}_{t_{0}}(H \Pi)\right)=\sum_{s=0}^{N-1} e^{-r\left(t_{s+1}-t_{s}\right)} \sum_{(j, l) \in K_{j s}} \sum_{m=j_{s}}^{j_{s+1}-1} \sum_{\left\{(k, i) \in H_{m}: A_{k, i} \subset A_{j, l}^{t_{s}}\right\}} \sqrt{P\left(A_{k, i}\right)}\left|\left\langle X, \psi_{k, i}\right\rangle\right| .
$$

Making use of the coarser inequality in (5.20), the fact that $\sum_{(j, l) \in K_{j s}} P\left(A_{j, l}^{t_{s}}\right)=1$ and $j_{0} \equiv 0$,

$$
\begin{aligned}
\mathbf{E}\left(\mathrm{VT}_{t_{0}}(H \Pi)\right) & =\sum_{s=0}^{N-1} e^{-r\left(t_{s+1}-t_{s}\right)} \sum_{(j, l) \in K_{j_{s}}}\left(\frac{\left(P\left(A_{j, l}^{t_{s}}\right)\right)^{1+\epsilon_{t_{s}}}}{1-2 \delta^{1+\epsilon_{t_{s}}}}+\| 1_{A_{j, l}^{t_{s}} D_{t_{s}, t_{s+1}} \|_{2}^{2}}\right) \\
& \leq \sum_{s=0}^{N-1}\left(\frac{\left(\delta^{1+\epsilon_{t_{s}}}\right)^{j_{s}}}{1-2 \delta^{1+\epsilon_{t_{s}}}}+\left\|D_{t_{s}, t_{s+1}}\right\|_{2}^{2}\right) \leq \frac{1}{\left(1-2 \delta^{1+\epsilon}\right)} \frac{1}{\left(1-\delta^{1+\epsilon}\right)}+\left\|\mathbf{E}\left(X \mid \mathcal{F}_{T}^{n}\right)-\mathbf{E}(X)\right\|_{2}^{2} .
\end{aligned}
$$

Remark 5.20. Notice how the summation over the time index is easily handled with a geometric sum resulting in $1 /\left(1-\delta^{1+\epsilon}\right)$ and a telescopic sum for the squared norms of the martingale differences. As we indicated, one may substantially improve the factor $1 /(1-$ $2 \delta^{1+\epsilon}$ ) by making use of finer bounds introduced in previous results. Numerical examples for volumes of transactions, their bounds and values of $\epsilon$ are displayed in Tables 1-6.

\section{Numerical Examples}

This section presents output from a computer implementation based on the Brownian motion example from Appendix A. This type of $H$-system can be seen to correspond to an initial partition corresponding to the recursive construction of Willinger (referred to in our Section 3), we have chosen such an example for matters of simplicity. Also, the fact that we have chosen to discretize the geometric Brownian model is also a matter of convenience, any other model with continuous paths and independent increments will also be possible (see the discussion after Theorem 4.6). Figures and tables with numerical outputs are presented in Tables 1-6 and Figures 1-6.

The simple discretization from Appendix A gives binary partitions $\mathcal{R}_{j}=\left\{A_{k, i}\right\}$ that are constructed via the increments of the Brownian motion and are characterized through the 


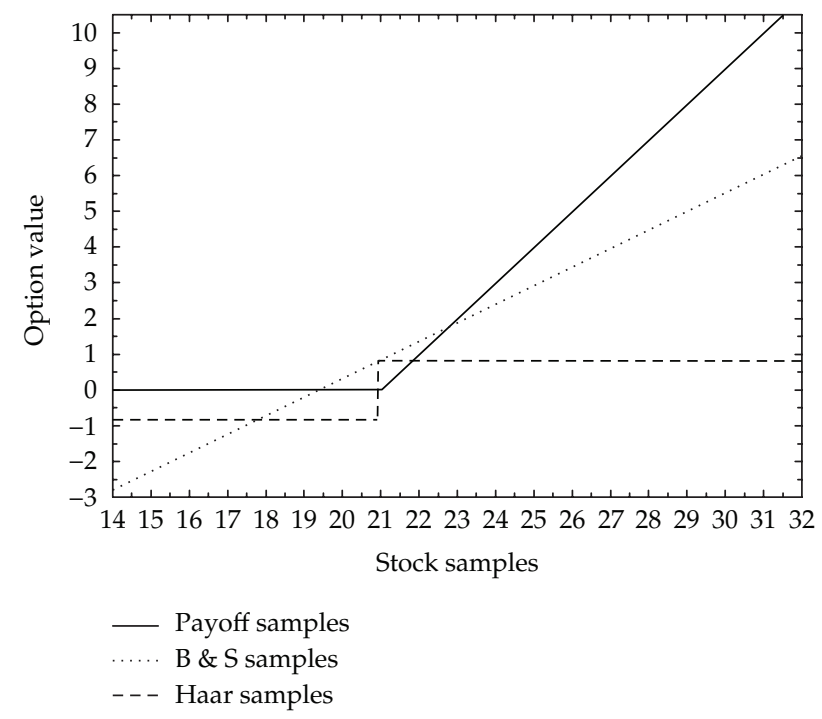

Figure 1: Approximations to single European call using delta hedging and a single Haar function (as explained in Section 6). Values of the parameters used: $N=1, S_{t_{0}}=20, r=0.05, \sigma=0.1, T-t_{0}=1, K=21$.

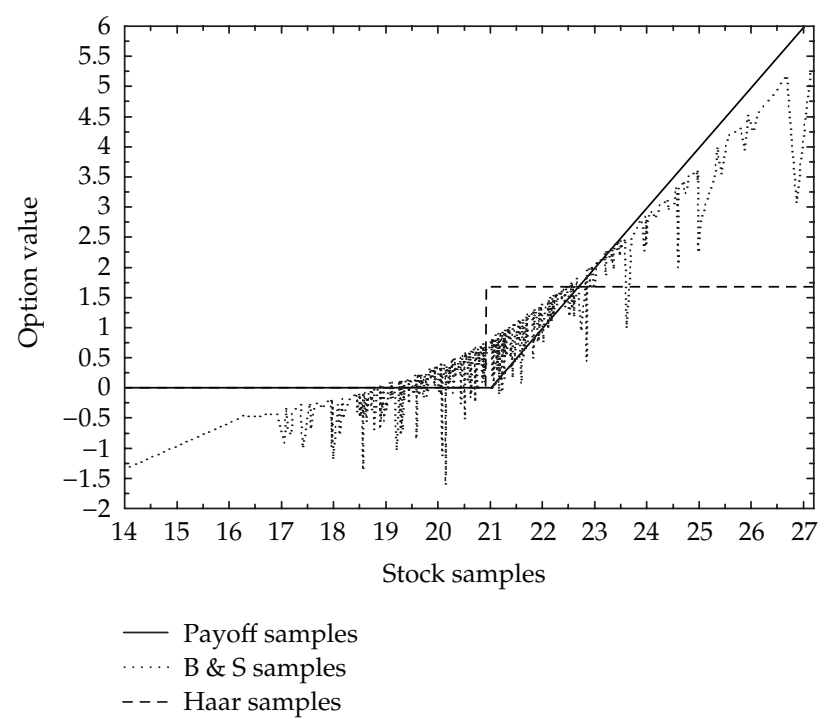

Figure 2: Same as in Figure 1 but with $u_{0}$ added.

Table 2: Volume of transactions for single European call, that is, option $Z_{1}$. Values of parameters as in Figure 1 , also, $J=16$ and $V_{t_{0}}(X)=0.809$.

\begin{tabular}{ccccccc}
\hline$R$ & $\widehat{\mathrm{VT}}(\Pi)$ & AverageVolTrBS & $\widehat{\mathrm{VT}}(H \Pi)$ & Fine bound & Coarse bound & Relative error \\
\hline$R=5$ & 0.920208 & 53.32 & 1.6783 & 1.93614 & 22.9151 & 0.227333 \\
$R=10$ & 0.95818 & 107.1 & 1.84845 & 2.36697 & 22.982 & 0.112873 \\
$R=15$ & 0.95818 & 157.2 & 1.89718 & 2.5557 & 22.9943 & 0.0746597 \\
$R=20$ & 0.959513 & 213.8 & 1.92295 & 2.70761 & 22.9983 & 0.0570624 \\
$R=25$ & 0.959513 & 258.8 & 1.94195 & 2.84482 & 23.0004 & 0.0447283 \\
$R=30$ & 0.959513 & 317 & 1.94998 & 2.90008 & 23.0015 & 0.0369233 \\
\hline
\end{tabular}




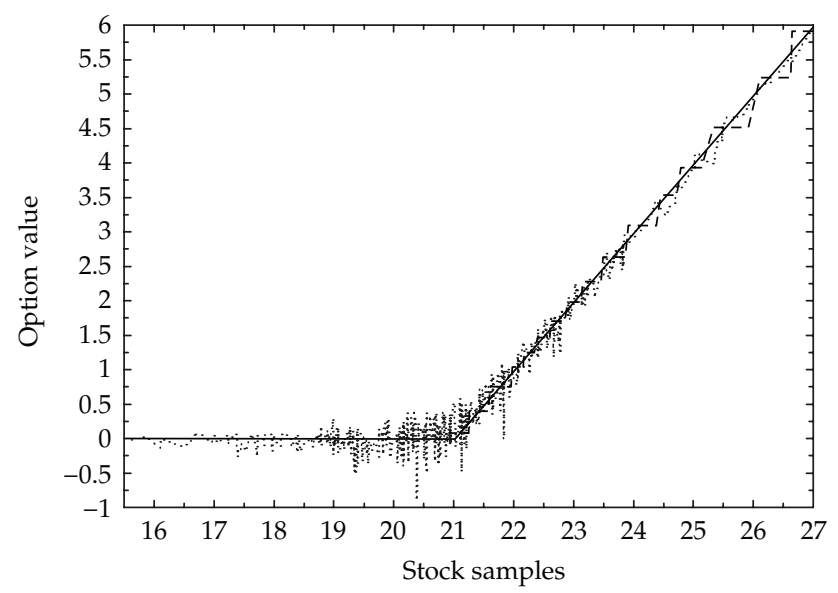

— Payoff samples

..... B \& $S$ samples

- - - Haar samples

Figure 3: Same as in Figure 1 except $R=20$.

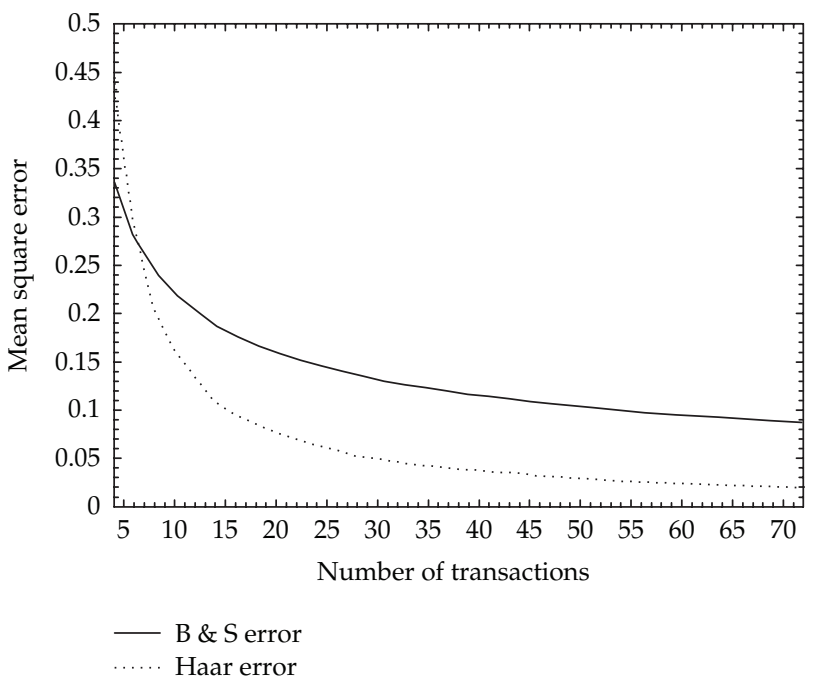

Figure 4: $L^{2}$ norm of the (relative) errors between the option $Z_{1}$ and delta hedging and Haar approximations respectively. The plot is in terms of the parameter $R$. Values of the parameters used: as in Figure 1.

parameters $N$ and $j_{1}, \ldots, j_{N}$. In this example all elements of $\mathcal{R}_{j}$ split in $\boldsymbol{R}_{j+1}$, therefore the indexation introduced in Section 2 gives for $0 \leq j \leq j_{N}$

$$
\begin{aligned}
\mathcal{R}_{j} & =\left\{A_{j, i}: 0 \leq i \leq 2^{j}-1\right\}, \\
K_{j} & =\left\{(j, i): 0 \leq i \leq 2^{j}-1\right\},
\end{aligned}
$$




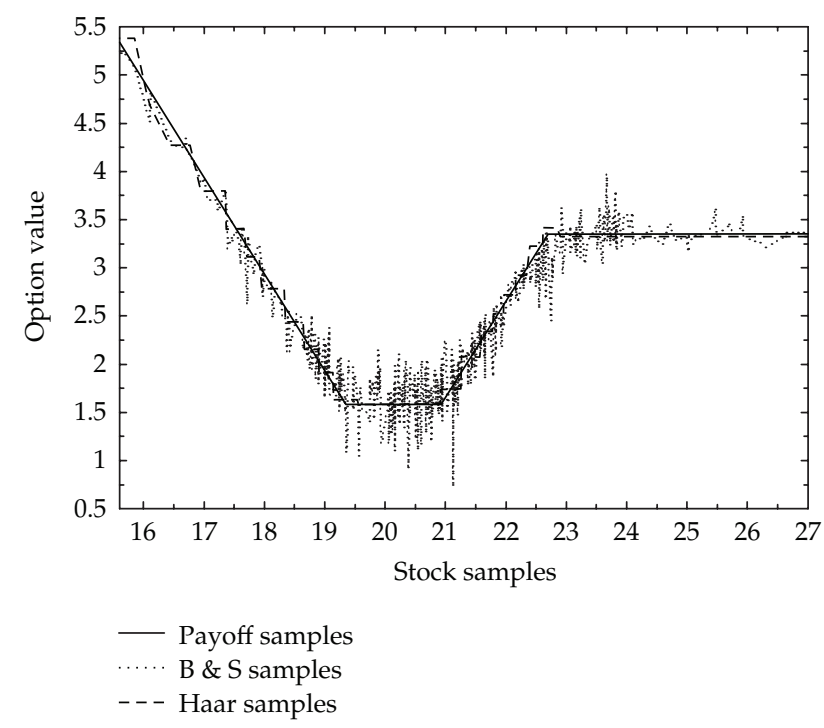

Figure 5: Approximations to portfolio $Z_{2}$, constructed from two calls and one put, using delta hedging and the Haar system constructed via Brownian motion increments. Values of the parameters used: $N=1$, $R=20, S_{t_{0}}=20, r=0.05, \sigma=0.1, T-t_{0}=s_{1}, K_{1}=19.35, K_{2}=20.96, K_{3}=22.7$.

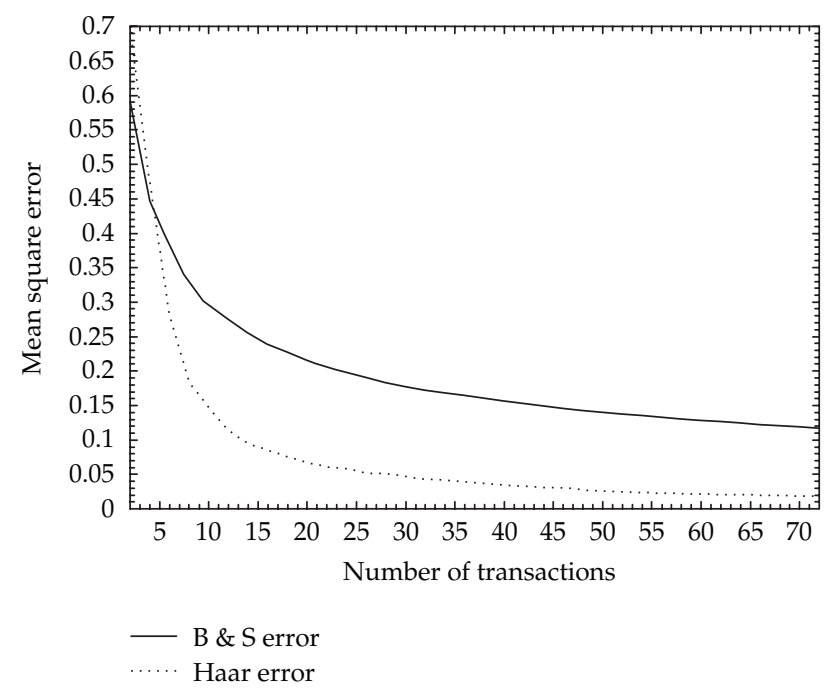

Figure 6: $L^{2}$ norm of the (relative) errors between the option $Z_{2}$ and delta hedging and Haar approximations, respectively. The plot is in terms of the parameter $R$. Values of the parameters used: as in Figure 5.

and $H_{j}=K_{j}$ for $0 \leq j<j_{N}$. Moreover,

$$
u_{0}=\mathbf{1}_{\Omega}, \quad\left\{u_{2^{j}+i} \equiv \psi_{j, i}: 0 \leq j<j_{N}, 0 \leq i \leq 2^{j}-1\right\} .
$$




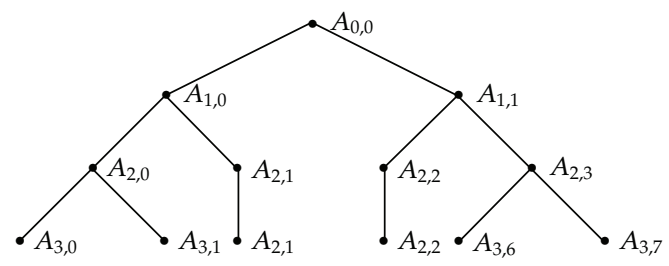

Figure 7

Table 3: Volume of transactions for portfolio composed of two calls and one put, that is, option $Z_{2}$. Values of parameters as in Figure 5, also, $J=14$ and $V_{t_{0}}(X)=2.3$.

\begin{tabular}{lcccccc}
\hline$R=R_{H}$ & $\widehat{V T}(B \Pi)$ & AverageVolTrBS & $\widehat{V T}(H \Pi)$ & Fine bound & Coarse bound & Rel. Error \\
\hline$R=5$ & 0.606359 & 27.1 & 1.04108 & 1.69357 & 7.42379 & 0.361129 \\
$R=10$ & 0.652564 & 59.43 & 1.21478 & 2.0247 & 8.12219 & 0.167056 \\
$R=15$ & 0.652564 & 90.75 & 1.26068 & 2.15734 & 8.89706 & 0.110117 \\
$R=20$ & 0.658014 & 121.59 & 1.28732 & 2.28512 & 8.89993 & 0.0861122 \\
$R=25$ & 0.658014 & 152.03 & 1.30466 & 2.40028 & 9.28114 & 0.0676239 \\
$R=30$ & 0.659379 & 184.9 & 1.31901 & 2.61102 & 9.64969 & 0.056536 \\
\hline
\end{tabular}

For the sake of simplicity we will take $r_{s} \equiv j_{s}-j_{s-1}=J$, for all $s=1, \ldots, N$, where $J$ is a fixed constant and $j_{0} \equiv 0$. Given the nature of our numerical experiments, there will be a natural way to select the value of $N$; the parameter $J$ is chosen empirically in such a way that (consider $n \equiv(N, J)$ )

$$
\left\|X-\mathrm{E}\left(X \mid F_{t_{N}}^{n}\right)\right\|_{2} \leq \eta
$$

for a negligible $\eta$. In our simulations we have taken $J=14,16$ for the static cases and $J=$ $5,8,10$ (depending on the option under consideration) for each stage in the dynamic cases.

We will make use of the notion of compression as described in Section 4.2 and some of the definitions and results introduced elsewhere in the paper.

For dynamic hedging, at each node $A_{j, l}^{t_{s-1}}$ of an unfolding path $w \in \cap A_{j_{s}, l_{s}}^{t_{s}} s=0, \ldots, N$, we will keep a constant (i.e., independent of the node) number $R_{H}$ of inner products $\left\langle X, \psi_{k, i}\right\rangle$ with $A_{k, i} \in \tau_{A_{j, 1}^{t_{s}-1}}$. Therefore, $R_{H}$ is the number of Haar obligations (see Section 4), purchased at $t_{s-1}$ and expiring at $t_{s}$, which are used by the portfolio $H \Pi$ when constructing $X_{R}^{c}$. Notice that, with this in mind, we then require $N \times R_{H}=R$. Considering $R_{H}$ to be constant helps to easy the comparisons with delta hedging but it is not a necessary constraint. In practice one may choose $R_{H}$ adaptively depending on the node in order to make sure a certain level of desired approximation is reached. Notice that static hedging corresponds to $N=1$ and so $R_{H}=R$.

To summarize, the parameter $R$ will be equal to the number of times the Black-Scholes portfolio is re-balanced when performing delta hedging and also equals the total number of Haar functions used in the final approximation when performing Haar hedging. $R$ will be referred to as the number of transactions in the remaining of the paper. We re-balance the Black-Scholes portfolio at uniformly spaced time intervals. We report the errors in the approximations as well as the volume of transactions, both as a function of the number of 
Table 4: Dynamic hedging information for option $Z_{1} . J=5$ and $R_{H}=5$ at each time step, remaining values of parameters as in Figure 1. The volume of transactions $\widehat{V T}(\cdot)$ are averages over all the samples.

\begin{tabular}{lcccccc}
\hline$R\left(R_{H}=5\right)$ & $\widehat{V T}(B \Pi)$ & $\widehat{V T}(H \Pi)$ & Fine bound & Coarse bound & Relative error & $\epsilon$ \\
\hline$R=10(N=2)$ & 1.26062 & 2.40553 & 3.73645 & 18.3088 & 0.0795891 & 0.0939396 \\
$R=15(N=3)$ & 1.47833 & 2.8358 & 4.46665 & 21.2139 & 0.0874086 & 0.0783706 \\
$R=20(N=4)$ & 1.63339 & 3.13998 & 5.08153 & 24.1135 & 0.09152 & 0.06748 \\
$R=25(N=5)$ & 1.8458 & 3.53484 & 5.50147 & 30.4139 & 0.0957869 & 0.0520525 \\
\hline
\end{tabular}

Table 5: Dynamic hedging information for the option $Z_{2} . J=5$ and $R_{H}=8$ at each time step, remaining values of parameters as in Figure 5. The volume of transactions $\widehat{V T}(\cdot)$ are averages over all the samples.

\begin{tabular}{ccccccc}
\hline$R\left(R_{H}=8\right)$ & $\widehat{V T}(B \Pi)$ & $\widehat{V T}(H \Pi)$ & Fine bound & Coarse bound & Relative error & $\epsilon$ \\
\hline$R=16(N=2)$ & 0.593357 & 1.20214 & 3.85979 & 49.1631 & 0.128758 & 0.0305896 \\
$R=24(N=3)$ & 1.03076 & 1.96901 & 4.39251 & 55.9105 & 0.128939 & 0.0268424 \\
$R=32(N=4)$ & 1.15317 & 2.2223 & 7.59714 & 77.4299 & 0.125603 & 0.0192216 \\
$R=40(N=5)$ & 1.37302 & 2.74642 & 9.13719 & 121.156 & 0.1235 & 0.0121686 \\
\hline
\end{tabular}

transactions. Equivalently, one may report these quantities as functions of the approximation error.

Whenever feasible, we report relative errors given by:

$$
\frac{\left\|X-X_{R}^{c}\right\|_{2}}{\|X\|_{2}}
$$

where, as usual, \|\|$_{2}$ is the $L^{2}$ norm. Given the nature of our computations we will use $\mathrm{E}\left(X \mid \mathcal{F}_{t_{N}}^{n}\right)$ instead of $X$ in (6.4), this gives a negligible error (see (6.3)). In order to reduce computational times, and only when doing dynamic hedging, we will report a sampled version of (6.4) (see (6.15)).

\subsection{Upper Bounds}

The exact values for the volume of transactions for the portfolio НП are given by the equalities in (5.24) and (5.29) for the static and dynamic, case respectively. We will also report the fine upper bound given by (5.25) (for the static case) and (5.30) (for the dynamic case). Analogously, we provide the coarse upper bound given by (5.26) (for the static case) and the term (5.31) (for the dynamic case). Notice that all these quantities refer to the pointwise volume of transactions (which, in the static case, equals its mean value). Also the parameter $\epsilon$ will be computed.

In practice, it is convenient to distinguish between two classes of type I of $A_{k, i}$ nodes (introduced in Definition 5.15), namely, the ones that satisfy the further constraint

$$
\left|\left\langle X, \psi_{k, i}\right\rangle\right|<P\left(A_{k, i}\right)^{1 / 2+k}
$$

For a relatively large $k$, we took $k=3$ in our simulations. This refinement reduces the values of the numbers $q_{m}$ and makes the values of the numbers $\epsilon_{m}$ and $\epsilon$ larger (and so makes the fine bounds tighter). These numbers appear in the inequalities of Corollary 5.17 and Theorem 5.18 
Table 6: Dynamic hedging information for the option $Z_{3}$. Values of parameters: $N_{A}=2, \ldots, 5, K_{A}=21$.

\begin{tabular}{lcccccc}
\hline$R\left(R_{H}=10\right)$ & $\widehat{V T}(B \Pi)$ & $\widehat{V T}(H \Pi)$ & Fine bound & Coarse bound & Relative error & $\epsilon$ \\
\hline$R=20(N=2)$ & 0.958413 & 1.66733 & 2.86227 & 8.78945 & 0.05 & 0.225927 \\
$R=30(N=3)$ & 1.01458 & 1.6126 & 2.93135 & 8.03383 & 0.0715974 & 0.245643 \\
$R=40(N=4)$ & 1.03078 & 1.53383 & 2.92057 & 7.8773 & 0.0740302 & 0.249264 \\
$R=50(N=5)$ & 1.22817 & 1.87943 & 3.1592 & 8.53391 & 0.0735656 & 0.225817 \\
\hline
\end{tabular}

The net effect on upper bounds of introducing the class of type I nodes satisfying (6.5) is an additive term with value no larger than 1.6. Our numerical simulations will report fine and coarse upper bounds including this modification.

\subsection{Multiresolution Analysis Algorithm}

The computations can be organized by means of the multiresolution analysis algorithm (MRA). The algorithm is an adaptation of the well-known algorithm from wavelet theory [20] to our probabilistic setting. This algorithm produces a relation between the samples of $X$, namely,

$$
x_{k}^{t_{s}}[i]=\mathrm{E}\left(X \mid \mathcal{F}_{t_{s}}^{n}\right)(w), \quad \omega \in A_{k, i}^{t_{s}} \in D_{t_{s}}^{n}
$$

and the Haar coefficient $\left\langle X, \psi_{k, i}\right\rangle$. This facilitates the computation and manipulation of these inner products. the algorithm for our setting is described in [14] (see also below).

Given that we will be reporting pathwise quantities, we have to consider (6.6) relative to a given node $A_{j, l}^{t_{s-1}}$, and therefore our algorithm consists of an unfolding path $w \in \cap A_{j_{s}, l_{s}}^{t_{s}}$ and, at each time step $t_{s}, s=0, \ldots, N-1$, the quantities $x_{k}^{t_{s}}[i]$ (given by (6.6)), conditioned to $A_{j_{s-1}, l_{s-1}}^{t_{s-1}}$, are inputs to the MRA algorithm described in [14]. Once the inner products $\left\langle X, \psi_{k, i}\right\rangle$ are available, we perform compression. After this, in order to compute the volume of transactions and the associated upper bounds, only the numbers $\left\langle X_{R}^{c}, \psi_{k, i}\right\rangle$ are needed.

The input to the MRA is obtained by computing

$$
x_{k}^{t_{s}}[i]=2^{j_{s}} \int_{A_{k, i}^{t_{s}}} X(\omega) d P(\omega), \quad(k, i) \in K_{j_{s}}
$$

where $P\left(A_{k, i}^{t_{s}}\right)=1 / 2^{j_{s}}$. Recalling that $r_{s}=j_{s}-j_{s-1}=J$, we observe that there are $2^{J}$ numbers in the array $x_{k}^{t_{s}}[\cdot]$.

For completeness, we include a sketch of the MRA, for the stage $s-1 \rightarrow s$. 
Analysis Step

Set $d_{k}[i] \equiv\left\langle X, \psi_{k, i},\right\rangle$

$$
\text { for } 1 \leq k \leq J, \quad x_{k}[i] \longrightarrow\left\{\begin{array}{l}
x_{k-1}[i]=\frac{1}{2}\left(x_{k}[2 i+1]+x_{k}[2 i]\right), \\
d_{k-1}[i]=2^{-\left(j_{s-1}+k+1\right) / 2}\left(x_{k}[2 i]-x_{k}[2 i+1]\right),
\end{array} \quad 0 \leq i \leq 2^{k-1}\right.
$$

\section{Compression Step}

For $0 \leq k \leq J-1$, keep the greatest $R$ coefficients $d_{k}^{\text {comp }}[i] \equiv d_{k}[i]$, and set $d_{k}^{\text {comp }}[i] \equiv 0$ for the rest of the $2^{J}-1-R$ ones.

\section{Reconstruction Step}

Consider $x_{0}^{\text {comp }}[0] \equiv x_{0}[0]$, for $k=1, \ldots J$,

$$
d_{k-1}^{\text {comp }}[i] \longrightarrow\left\{\begin{array}{c}
x_{k}^{\text {comp }}[2 i]=x_{k-1}^{\text {comp }}[i]+2^{\left(j_{s-1}+k-1\right) / 2} d_{k-1}^{\text {comp }}[i], \\
x_{k}^{\text {comp }}[2 i+1]=x_{k-1}^{\text {comp }}[i]-2^{\left(j_{s-1}+k-1\right) / 2} d_{k-1}^{\text {comp }}[i]
\end{array}\right.
$$

Some details on how to compute $x_{k}^{t_{s}}[i]$ are provided later in this section.

\subsection{Delta Hedging}

Delta hedging will be performed over $R$ time steps. Letting

$$
\varphi_{t_{i}}=\frac{\partial V_{t_{i}}(X)}{\partial S_{t_{i}}}
$$

the volume of transactions for a Black-Scholes portfolio with rebalancing dates $\left\{t_{i}\right\}, i=$ $0, \ldots, R-1$ is given by

$$
\sum_{i=0}^{R-1}\left[\left|\varphi_{t_{i}}-\varphi_{t_{i-1}}\right| S_{t_{i}}+\left(B_{t_{i}}-B_{t_{i-1}} e^{r\left(t_{i}-t_{i-1}\right)}\right)_{+}\right]
$$

with $\varphi_{t_{-1}}=B_{t_{-1}} \equiv 0$ ( $B_{t}$ is the bond used in Section 4.1$)$. We have used equally spaced rebalancing dates. Given that (6.11) is a random quantity, we will report the average (AverageVolTrBS) over many samples.

\subsection{Path Independent Options}

For the path independent examples we will consider two cases: (i) $N=1$ which provides static hedging, in this case all the atomic sigma algebras $\mathscr{A}_{n}$ are included in $\sigma\left(W_{T}\right)$ and $\mathcal{A}_{\infty}=\sigma\left(W_{T}\right)$; (ii) $N>1$ which provides dynamic hedging. Mathematically, in the case of 
path independent options, already the case $N=1$ provides perfect replication in the limit of $J \rightarrow \infty$.

\section{(i) Static Case}

The expressions for the pathwise volume of transactions for our portfolios in this case are

$$
\begin{gathered}
\widehat{\mathrm{VT}}_{t_{0}}(B \Pi)=e^{-r\left(T-t_{0}\right)}\left\|X_{R}^{c}\right\|_{1}=e^{-r\left(T-t_{0}\right)} \int_{\Omega}\left|X_{R}^{c}(\omega)\right| d P(\omega), \\
\widehat{\mathrm{VT}}_{t_{0}}(H \Pi)=e^{-r\left(T-t_{0}\right)} \sum_{A_{k, i} \in \mathcal{C}_{\Omega}} \sqrt{P\left(A_{k, i}\right)}\left|\left\langle X_{R}^{c}, \psi_{k, i}\right\rangle\right| .
\end{gathered}
$$

Next we comment on the output displays (see Tables 1-6 and Figures 1-6); consider first a single European call $Z_{1}=\left(S_{T}-K\right)_{+}$, values of parameters are indicated in the text surrounding the figures. In Figures 1, 2, and 3 we present the Black-Scholes and Haar approximations with $R=1,2,20$, respectively. Notice how Figure 1 shows the Haar approximation with $u_{1}=1 / 2\left(1_{A_{1,0}}-1_{A_{1,1}}\right)$, which happens to give the largest inner product. Figure 2 shows the Haar approximation when $u_{0}$ is added, giving the second largest inner product in this example. Figure 4 shows the estimation of the $L^{2}$ norm of the errors as a function of $R$. Similar information is presented in Table 1; it illustrates the compression power of our $H$-system for the call option $Z_{1}$ in the present static case. It shows that few Haar functions are needed to implement $Н \Pi$. That table provides the results for different values of $J$ as this better illustrates the effect of the optimization carried out in (4.13).

As a second example we consider a portfolio built as a linear combination of European calls and puts as follows, $Z_{2}=\left(S_{T}-K_{1}\right)_{+}+\left(K_{2}-S_{T}\right)_{+}-\left(S_{T}-K_{3}\right)+$, values of parameters are indicated in the text surrounding Figure 5. Finally, Figure 6 shows the estimation of the $L^{2}$ norm of the errors as a function of $R$.

Tables 2 and 3 show the volume of transactions for the portfolio $B \Pi$, for delta hedging and for the Haar portfolio $Н П$. The tables also contain values of the fine and coarse upper bounds for the portfolio $H \Pi$.

Notice the difference in magnitudes with AverageVolTrBS. The volume of transactions offers a clear numerical evidence of the different nature of Haar hedging and delta hedging, an analytical analysis was provided in Section 5.

It is expected, and it is confirmed by our experience with numerical examples, that the Haar approximation outperforms (in the sense of smaller error for equal value of $R$ ) the Black-Scholes approximation whenever the payoff, or its derivative, contains discontinuities. Moreover, the Haar functions can be adapted to these discontinuities; for instance, we can choose $u_{1}$ such that it is supported in the union of $A_{1,0}=\left\{S_{T}<K\right\}$ and $A_{1,1}=\left\{S_{T} \geq K\right\}$ for the case of the European call. Our examples reflect these choices; for example, $S_{t_{0}}$ was taken close to $K$ so as the discontinuity in the first derivative of the European call becomes problematic for Black-Scholes approximation and can be reproduced efficiently by the Haar expansion. An extreme example of this kind will be the case of a digital option where, of course, the Haar expansions have no bearing as a hedging tool.

Naturally, it is easy to find situations where delta hedging outperforms Haar hedging as, for example, a position in a European call which is well in or out of the money. This is a situation where the linear approximation in delta hedging becomes very efficient. It is 
interesting to see under what conditions delta hedging and Haar hedging are complementary and to investigate how to combine both techniques.

Tables 2 and 3 show that the behaviors of the portfolios $B \Pi$ and $H \Pi$ are similar in these static examples. It is also important to notice that we were able to make an effective use of the notion of compression because of the Haar representation; expansions on characteristic functions do not allow such a simple effective optimization procedure.

\section{(ii) Dynamic Case}

Given a path $w \in \cap_{s=0}^{N} A_{j_{s}, l_{s}}^{t_{s}}$, the volume of transactions for our portfolios is given by:

$$
\begin{aligned}
& \widehat{\mathrm{VT}}_{t_{0}}(B \Pi)(w)=\sum_{s=0}^{N-1} e^{-r\left(t_{s+1}-t_{s}\right)} \mathbf{1}_{A_{j_{s}, l_{s}}^{t_{s}}} \sum_{A_{k, i}^{t_{s+1}} \in \mathcal{\tau}_{A_{j_{s}, l_{s}}^{t_{s}}}} \frac{P\left(A_{k, i}^{t_{s+1}}\right)}{P\left(A_{j_{s}, l_{s}}^{t_{s}}\right)} \\
& \times\left|\left[\frac{1}{P\left(A_{k, i}^{t_{s+1}}\right)} \int_{A_{k, i}^{t_{s}+1}} X-\frac{1}{P\left(A_{j_{s}, l_{s}}^{t_{s}}\right)} \int_{A_{j_{s, l}, l_{s}}^{t_{s}}} X\right]\right|,
\end{aligned}
$$

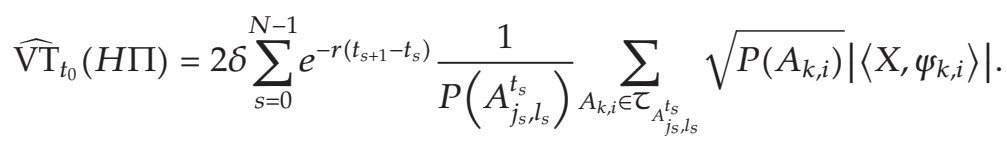

In practice we do not reproduce $D_{t_{s}, t_{s+1}}$ but its compressed version, and therefore the averages in (6.13) have to be replaced by the expressions described in the MRA above. Similarly the inner products in (6.14) have to be replaced by their compressed versions. An expression for the input to the MRA, at each dynamic stage, is provided by (6.18).

We illustrate the dynamic case for the path independent options $Z_{1}$ and $Z_{2}$ described above. Notice that our main point is to illustrate how the volume of transactions for the portfolios $B \Pi$ and $H \Pi$ behaves when transactions are performed over time, that is in a dynamic way. Naturally, we will report average values (over $M$ random paths, we took $M$ between 200 and 500) of all the quantities involved: volume of transactions, fine and coarse bounds, relative errors, and the values of $\epsilon$. For example, if we sample $w_{1}, \ldots, w_{M}$ paths, the absolute error becomes

$$
\frac{1}{M} \sum_{m=1}^{M}\left|X\left(w_{i}\right)-X_{R}^{c}\left(w_{i}\right)\right|^{2}
$$

Tables 4 and 5 display the resulting values averaged between $M=200$ and $M=500$ random paths for the options $Z_{1}$ and $Z_{2}$, respectively. The bounds correspond to the portfolio $Н \Pi$. Noticing the need to use the parameter $R_{H}$, for simplicity we fixed this parameter to be $R_{H}=5$ for $Z_{1}$ and $R_{H}=8$ for $Z_{2}$ to reduce the error and increased $N$ (and so also increased $\left.R=R_{H} \times N\right)$. One may also work with an adaptive value of $R_{H}$. As indicated, we are reporting averages of $\widehat{\mathrm{VT}}(\cdot)$ over all the samples, the oscillations around these values seem to be negligible.

Another illustration is provided by a path dependent example, it necessarily requires dynamic hedging. Again, our main objective is to illustrate how the volume of transactions 
for both of our portfolios behaves when performing transactions over time. We consider the Asian option

$$
Z_{3}=\left(\frac{1}{N_{A}} \sum_{s=0}^{N_{A}-1} S_{t_{N_{A}-s}}-K_{A}\right)
$$

It is natural to deploy the $H$-system along the required times, $t_{0}, t_{1}, \ldots, t_{N_{A}}$ and so we will take $N=N_{A}$.

For each sampled path, the computation of $x_{k}^{t_{s}}[i]$ involves $N$ iterated integrals. Indeed, the stock, discretized in time, follows an evolution $S=F\left(W_{t_{0}, t_{1}}, \ldots, W_{t_{N-1}, t_{N}}\right)$ where $\left\{W_{t_{s-1}, t_{s}}\right\}_{s=1}^{N}$ are independent random variables with normal distribution, and then the atoms of $\mathcal{F}_{t_{s}}^{n}$ contained in $A_{k_{s-1}, i_{s-1}}^{t_{s-1}}$ are given by

$$
A_{k, i}^{t_{s}}=W_{t_{s-1}, t_{s}}^{-1}\left(\left(c_{i}^{J}, c_{i+1}^{J}\right]\right) \cap W_{t_{s}, t_{s+1}}^{-1}((-\infty, \infty)) \cap \ldots, \cap W_{t_{N-1}, t_{N}}^{-1}((-\infty, \infty)),
$$

where the $c_{i}^{J}$ are given in Appendix A. Consequently

$$
x_{k}^{t_{s}}[i]=2^{j_{s}} \int_{-\infty}^{\infty} \cdots \int_{-\infty}^{\infty} \int_{c_{i}^{J}}^{c_{i+1}^{J}} \int_{c_{i_{s-1}}^{J}}^{c_{i_{s-1}+1}^{J}} \cdots \int_{c_{i_{1}}^{J}}^{c_{i_{1}+1}^{J}}\left(F\left(y_{1}, \ldots, y_{N}\right)-K_{A}\right)_{+} e^{-y_{1}^{2} / 2} \cdots e^{-y_{N}^{2} / 2} d y_{1} \cdots d y_{N} .
$$

In order to avoid exceedingly long computational times we have taken $N_{A}=2, \ldots, 5$. Notice that in order to reduce the error of approximation we are required to increase $R_{H}$ (we have taken $R_{H}=10$ ). Table 6 displays the resulting values averaged over approximately $M=500$ random paths.

\section{Conclusions and Extensions}

We have introduced a basic and general new framework to represent contingent claims. Key ingredients are the flexibility given by the possible space and time discretizations that can be adapted to a given class of options and the potential for financial realization of these discretizations. From a theoretical point of view, the approach is as fundamental as delta hedging and it is reasonable to think that it can be extended to other settings where this last technique is available. Some of the computational tools introduced could also be used even when an actual financial realization (of the approximation) is not available, pricing computations is an example. A main goal of the paper is to bring forward the importance of efficient hedging strategies, these alternative representations should be fully contrasted with delta hedging in order to compare trade-offs and limitations of this last technique. To this end, the paper emphasizes efficiency in relation to volume of transactions and gives theoretical as well as numerical evidence that the suggested new hedging strategy has several advantages over delta hedging.

Further empirical and theoretical work is needed to assess the realm of applications where the new constructions offer a financial or computational advantage. The techniques could also be extended to the setting of higher-dimensional models. 


\section{Appendices}

\section{A. Example: An H-System in the Black-Sholes Model}

The example describes how to construct a basic class of Haar systems associated to the BlackScholes model. The underlying process for the Black-Scholes model is a Brownian motion defined on a probability space $(\Omega, \mathcal{F}, Q)$ with filtration $\left(\mathcal{F}_{t}\right)_{t_{0} \leq t \leq T}$. The splitting of atoms will be performed using the Brownian motion increments. The price process under the risk neutral measure $P$ is given by $S_{t}: \Omega \rightarrow \mathbb{R}, t_{0} \leq t \leq T$,

$$
S_{t}(\omega)=S_{t_{0}} \exp \left(v\left(t-t_{0}\right)+\sigma \sqrt{\left(t-t_{0}\right)} W_{t}(\omega)\right)
$$

where $v=\left(r-\sigma^{2} / 2\right)$, and we have used the Gaussian random variables $W_{t}: \mathcal{N}(0,1)$ that are defined on $\left(\Omega, \Psi_{t}, P\right)$.

The construction will be based on two parameters; the first parameter $N$ will turn out to be the number of transaction dates during the period $\left[t_{0}, T\right]$ (see Section 6) and the second set of parameters $j_{1}, \ldots, j_{N}$ will be the scale or space discretizations associated with each trading date. For simplicity, the splitting of atoms will be in pieces of equal probability, this constrain can be easily removed. It is convenient to introduce first a "purely static" Haar system, considering $N=1$, which is applicable to path independent European options. This system will be the building block for the more general construction with $N \geq 1$. Therefore, we first concentrate on the sigma algebra $\sigma\left(S_{T}\right)=S_{T}^{-1}(\mathbb{B}(0, \infty))$, due to $\sigma\left(S_{T}\right)=\sigma\left(S_{T}^{-1}\left(\left(a_{1}, a_{2}\right]\right), 0<a_{1}<a_{2}<\infty\right)$. the following equation specifies $P$ on $\sigma\left(S_{T}\right)$, letting $B=S_{T}^{-1}\left(\left(a_{1}, a_{2}\right)\right)$ :

$$
P(B)=\frac{1}{\sigma \sqrt{2 \pi\left(T-t_{0}\right)}} \int_{a_{1}}^{a_{2}} \exp \left[\frac{-\left(\ln \left(s / S_{t_{0}}\right)-v\left(T-t_{0}\right)\right)^{2}}{2 \sigma^{2}\left(T-t_{0}\right)}\right] \frac{d s}{s} .
$$

From our previous notation, $W_{T}: \Omega \rightarrow \mathbb{R}$

$$
P\left(W_{T}^{-1}(A)\right)=\frac{1}{\sqrt{2 \pi}} \int_{A} e^{-y^{2} / 2} d y
$$

for any Borel subset $A \subset \mathbb{R}$. This equation gives $P$ on $\sigma\left(W_{T}\right)=W_{T}^{-1}(\mathbb{B}(\mathbb{R})) \subseteq \mathcal{F}_{T}$. Clearly, $\sigma\left(S_{T}\right)=\sigma\left(W_{T}\right)$. Denote the cumulative standard normal distribution by

$$
\Phi(z)=\frac{1}{\sqrt{2 \pi}} \int_{-\infty}^{z} e^{-y^{2} / 2} d y
$$

Given an integer $j$, define the numbers $-\infty=c_{0}^{j}<c_{1}^{j}<\cdots<c_{2^{j}}^{j}=\infty$ such that

$$
\Phi\left(c_{i+1}^{j}\right)-\Phi\left(c_{i}^{j}\right)=\frac{1}{2^{j}}, \quad \forall i=0, \ldots, 2^{j}-1
$$


Whenever encountered, the inequality $\leq \infty$ should be interpreted to mean $<\infty$. We define the binary splitting of atoms inductively by setting $A_{0,0}=\Omega$, and for given $j$ consider $0 \leq i \leq 2^{j}-1$,

$$
\begin{aligned}
A_{j+1,2 i} & =\left\{w \in A_{j, i} \mid c_{2 i}^{j+1}<W_{T}(\omega) \leq c_{2 i+1}^{j+1}\right\}, \\
A_{j+1,2 i+1} & =\left\{w \in A_{j, i} \mid c_{2 i+1}^{j+1}<W_{T}(\omega) \leq c_{2 i+2}^{j+1}\right\} .
\end{aligned}
$$

Note that $A_{j, i}=A_{j+1,2 i} \cup A_{j+1,2 i+1}$. Therefore we have defined a dyadic sequence of partitions $D=\left\{D_{j}\right\}_{j \geq 0}$ with $p_{j}=\left\{A_{j, i}\right\}, i=0, \ldots, 2^{j}-1$, where the atoms satisfy

$$
P\left(A_{j, i}\right)=\frac{1}{2^{j}}
$$

Setting $m=2^{j}$ and $\mathscr{A}_{m}=\sigma\left(\left\{A_{j, i}: i=0, \ldots, m-1\right\}\right)$ gives $\mathscr{A}_{\infty}=\sigma\left(\cup_{m \geq 0} \mathcal{A}_{m}\right)=\sigma\left(S_{T}\right)$. Notice that the above atoms correspond to partitioning the range of $S_{T}$.

It follows from Theorem 2.6 that there is a Haar system capable of approximating any random variable in $L^{2}\left(\Omega, \sigma\left(S_{T}\right), P\right)$, choosing a sufficiently large $J$.

We are now ready to describe the construction of a finite Haar system for an arbitrary $N \geq 1$. The idea is simply to construct a Haar dyadic system by a concatenation of several Haar systems, each of them analogous to the case $N=1$ but this later one is now restricted to smaller time intervals. Given an arbitrary sequence of times $t_{0}=t_{0}<t_{1}<\cdots<t_{N-1}<t_{N}=T$, we consider the Brownian motion increments $\sqrt{t_{i+1}-t_{i}} W_{t_{i}, t_{i+1}}$ where the random variables $W_{t_{i}, t_{i+1}} \sim \mathcal{N}(0,1)$ are independent. Fix a corresponding sequence of scales $\left\{j_{i}=j_{t_{i}}\right\}_{i=1}^{N}$, we will define the splitting of atoms on stages according to the time intervals $\left\{t_{i}, t_{i+1}\right\}$. For the first stage $\left\{t_{0}, t_{1}\right\}$ we define the binary splitting of atoms inductively by setting $A_{0,0}=\Omega$ and for $0 \leq j<j_{1}, i=0, \ldots, 2^{j}-1, A_{j+1, i}$ as in (A.6), using $W_{t_{0}, t_{1}}$ instead of $W_{T}$.

For the generic stage $\left\{t_{k}, t_{k+1}\right\}$, consider $j_{k} \leq j<j_{k+1}$ and $i=0, \ldots, 2^{j}-1$ as usual, let $p$ and $0 \leq q<2^{j+1-j_{k}}$ be, respectively, the quotient and residue in the integer division of $i$ by $2^{j+1-j_{k}}$, and then define inductively the sets

$$
\begin{gathered}
A_{j+1,2 i}=\left\{w \in A_{j, i}: c_{2 q}^{j+1-j_{k}}<W_{t_{k}, t_{k+1}} \leq c_{2 q+1}^{j+1-j_{k}}\right\}, \\
A_{j+1,2 i+1}=\left\{w \in A_{j, i}: c_{2 q+1}^{j+1-j_{k}}<W_{t_{k}, t_{k+1}} \leq c_{2 q+2}^{j+1-j_{1}}\right\} .
\end{gathered}
$$

\section{Acknowledgments}

The research of P. J. Catuogno is supported in part by a FAPESP grant. The research of S. E. Ferrando is supported in part by an NSERC grant.

\section{References}

[1] R. A. DeVore, "Nolinear approximation," Acta Numerica, vol. 7, pp. 51-150, 1998.

[2] D. L. Donoho, M. Vetterli, R. A. DeVore, and I. Daubechies, "Data compression and harmonic analysis," IEEE Transactions on Information Theory, vol. 44, no. 6, pp. 2435-2476, 1998.

[3] S. Figlewski, "Options arbitrage in imperfect markets," The Journal of Finance, vol. 44, pp. 1289-1311, 1989. 
[4] C. Albanese and S. Tompaidis, "Small transaction cost asymptotics and dynamic hedging," European Journal of Operational Research, vol. 185, no. 3, pp. 1404-1414, 2008.

[5] S. Mallat, A Wavelet Tour of Signal Processing, Academic Press, San Diego, Calif, USA, 2nd edition, 1998.

[6] D. L. Donoho, "Unconditional bases are optimal bases for data compression and for statistical estimation," Applied and Computational Harmonic Analysis, vol. 1, no. 1, pp. 100-115, 1993.

[7] D. L. Donoho, "Unconditional bases and bit-level compression," Applied and Computational Harmonic Analysis, vol. 3, no. 4, pp. 388-392, 1996.

[8] M. Avellaneda and A. Paras, "Managing the volatility risk of portfolios of derivative securities: the Lagrangian uncertain volatility model," Applied Mathematical Finance, vol. 3, pp. 21-52, 1996.

[9] E. Derman, D. Ergener, and I. Kani, “Static options replication," The Journal of Derivatives, vol. 2, no. 4, pp. 78-95, 1995.

[10] D. G. Hobson, "Robust hedging of the lookback option," Finance and Stochastics, vol. 2, pp. 329-347, 1998.

[11] A. Pechtl, “Classified information," RISK, vol. 8, no. 6, pp. 59-61, 1995.

[12] R. F. Gundy, "Martingale theory and pointwise convergence of certain orthogonal series," Transactions of the American Mathematical Society, vol. 124, pp. 228-248, 1966.

[13] J. Neveu, Discrete-Parameter Martingales, North-Holland, Amsterdam, The Netherlands, 1975.

[14] P. J. Catuogno, S. E. Ferrando, and A. L. Gonzalez, "Adaptive martingale approximations," The Journal of Fourier Analysis and Applications, vol. 14, no. 5-6, pp. 712-743, 2008.

[15] M. Girardi and W. Sweldens, "A new class of unbalanced Haar wavelets that form an unconditional basis for $L_{p}$ on general measure spaces," The Journal of Fourier Analysis and Applications, vol. 3, no. 4, pp. 457-474, 1997.

[16] W. Willinger, Pathwise Stochastic Integration and Almost-Sure Aproximation of Stochastic Processes [M.S. thesis], Cornell University, 1987.

[17] W. Willinger and M. S. Taqqu, "Toward a convergence theory for continuous stochastic securities market models," Mathematical Finance, vol. 1, no. 1, pp. 55-99, 1991.

[18] N. H. Bingham and R. Kiesel, Risk-Neutral Valuation, Pricing and Hedging of Financial Derivatives., Springer, London, UK, 1998.

[19] W. F. Stout, Almost Sure Convergence, Academic Press, 1974.

[20] S. G. Mallat, "Multiresolution approximations and wavelet orthonormal bases of $L^{2}(\mathbb{R})$," Transactions of the American Mathematical Society, vol. 315, no. 1, pp. 69-87, 1989. 


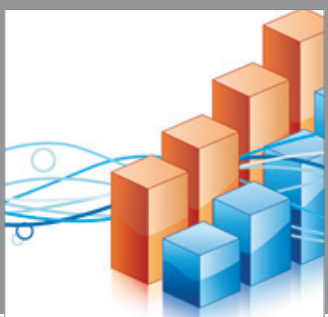

Advances in

Operations Research

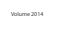

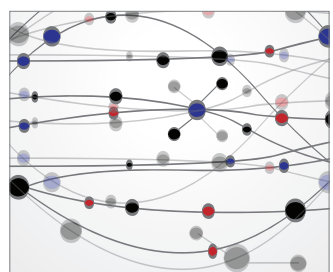

\section{The Scientific} World Journal
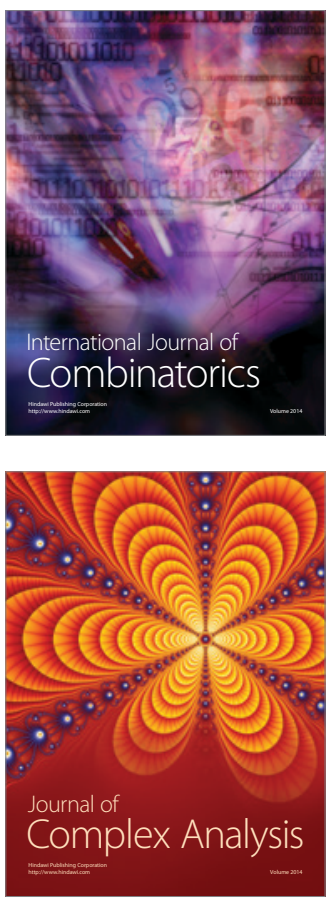

International Journal of

Mathematics and

Mathematical

Sciences
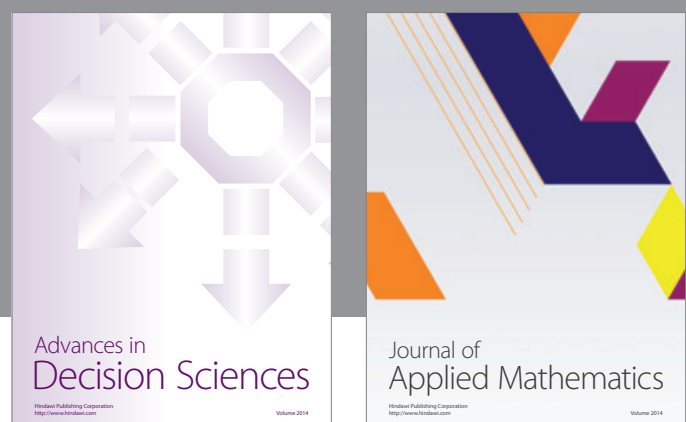

Journal of

Applied Mathematics
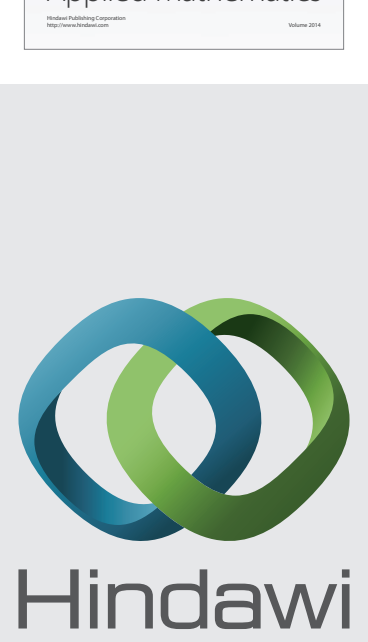

Submit your manuscripts at http://www.hindawi.com
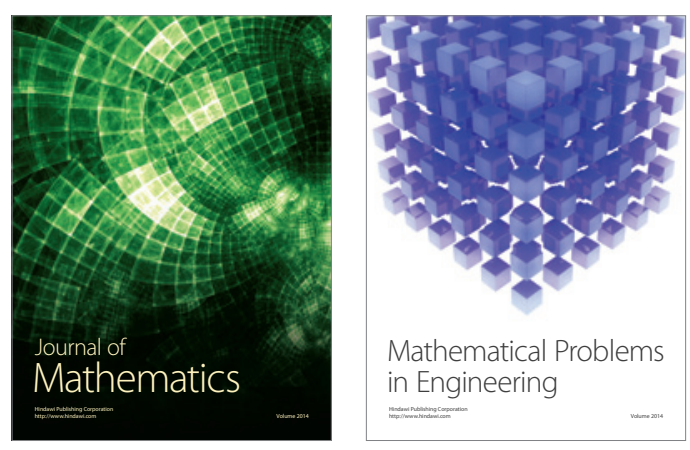

Mathematical Problems in Engineering
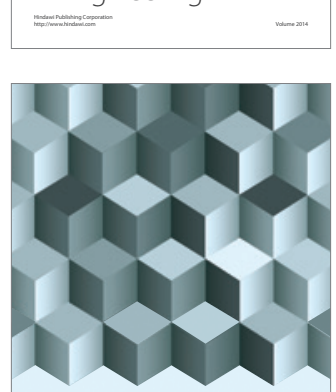

Journal of

Function Spaces
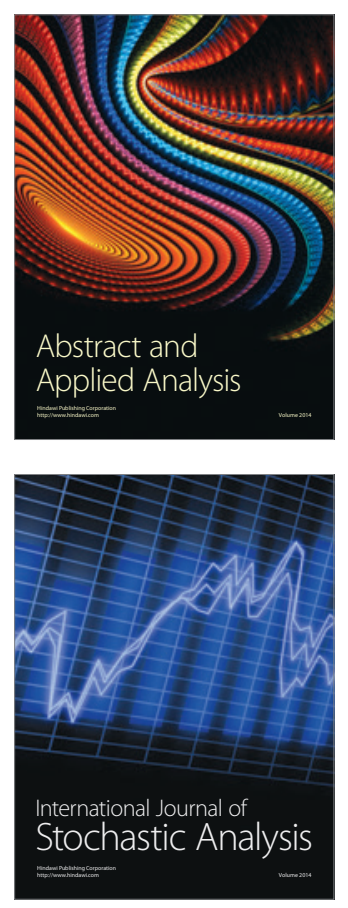

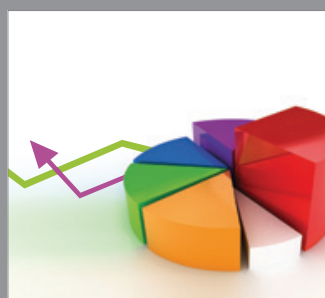

ournal of

Probability and Statistics

Promensencen
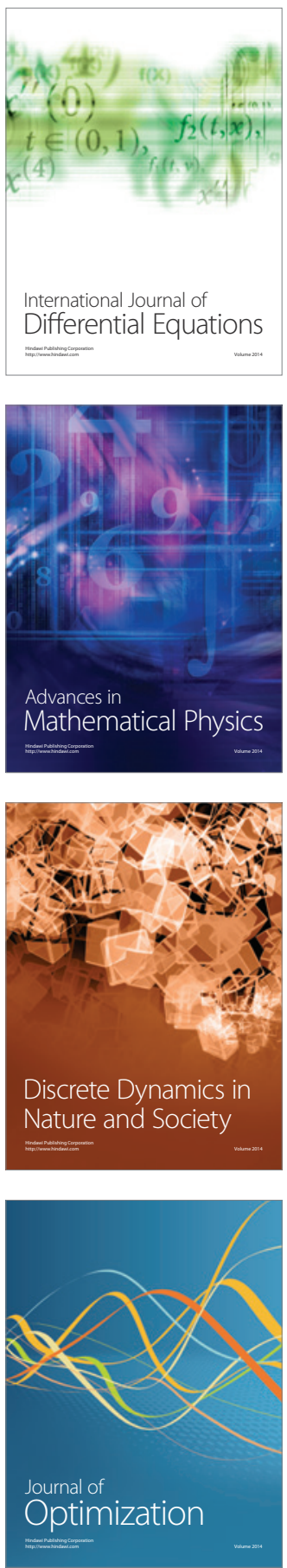